\title{
DIx1/2 mice have abnormal enteric nervous system function
}

\author{
Christina M. Wright, , James P. Garifallou, ${ }^{2,3}$ Sabine Schneider, ${ }^{1,3}$ Heather L. Mentch, ${ }^{1,3}$ \\ Deepika R. Kothakapa, ${ }^{1,3}$ Beth A. Maguire, ${ }^{1,3}$ and Robert O. Heuckeroth ${ }^{1,3}$ \\ 'Department of Pediatrics, ${ }^{2}$ Center for Applied Genomics, and ${ }^{3}$ Leonard and Madlyn Abramson Pediatric Research \\ Center, Children's Hospital of Philadelphia Research Institute and the Perelman School of Medicine at the University of \\ Pennsylvania, Philadelphia, Pennsylvania, USA.
}

Decades ago, investigators reported that mice lacking DLX1 and DLX2, transcription factors expressed in the enteric nervous system (ENS), die with possible bowel motility problems. These problems were never fully elucidated. We found that mice lacking DLX1 and DLX2 (DIx1/2 $2^{-1-}$ mice) had slower small bowel transit and reduced or absent neurally mediated contraction

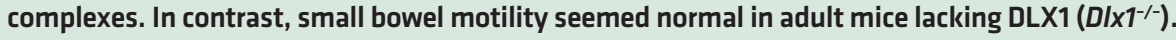
Even with detailed anatomic studies, we found no defects in ENS precursor migration, or

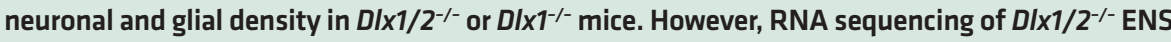
revealed dysregulation of many genes, including vasoactive intestinal peptide (Vip). Using immunohistochemistry and reporter mice, we then found that $D I x 1 / 2^{-/-}$mice have reduced VIP expression and fewer VIP-lineage neurons in their ENS. Our study reveals what we believe is a novel connection between DIx genes and Vip and highlights the observation that dangerous bowel motility problems can occur in the absence of easily identifiable ENS structural defects. These findings may be relevant for disorders like chronic intestinal pseudo-obstruction (CIPO) syndrome.

Conflict of interest: The authors declare that no conflict of interest exists.

Copyright: (ㄷ) 2020, American Society for Clinical Investigation.

Submitted: July 8, 2019

Accepted: January 22, 2020

Published: February 27, 2020

Reference information: /CI Insight. 2020;5(4):e131494.

https://doi.org/10.1172/jici.

insight.131494.

\section{Introduction}

Chronic intestinal pseudo-obstruction (CIPO) is a serious digestive disorder characterized by profound bowel motility defects, leading to severe constipation, abdominal distention, and life-threatening malnutrition requiring total parenteral nutrition (TPN). Although rare, CIPO is a leading cause of intestinal failure (1), accounting for approximately $10 \%$ to $14 \%$ of small intestine (SI) transplants (2). Many CIPO cases are believed to be caused by defects in the enteric nervous system (ENS), a complex network of neurons and glia within the bowel wall (3). The ENS develops from enteric neural crest-derived cells (ENCDCs) that migrate through the bowel during fetal development (4). ENCDCs give rise to diverse neuronal and glial subtypes that control nutrient absorption, epithelial secretion, intestinal mixing, and (critically) transport of contents down the bowel (5). In neuropathic CIPO, neurons and glia are present, but damaged or dysfunctional, leading to dysmotility. Neuronal dysfunction in CIPO has many etiologies, including infection, autoimmune disease, neurodegeneration, and gene mutations $(1,6)$. Genetic causes of CIPO are poorly understood. Although genes like SOX10 and POLG have been linked to neuropathic CIPO $(7,8)$, we believe many CIPOlinked genes remain to be discovered (9). Here, we examine the role of $D l x 1$ and $D l x 2$, two transcription factors expressed in developing ENS. We discovered that loss of these genes in mice causes profound intestinal dysmotility despite nearly normal ENS anatomy.

Dlx1 and Dlx2 are highly conserved homeobox transcription factors located in a tail-to-tail configuration on mouse chromosome 2 . They are essential for craniofacial, palate (10), tooth (11), and central nervous system (CNS) morphogenesis $(10,12-14)$. In the CNS, Dlx1 and Dlx2 are critical for subpallial

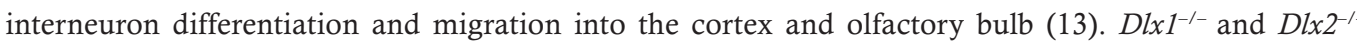
mice exhibit milder defects than $D l x 1 / 2$ double mutants, implying functional redundancy for DLX1 and DLX2 in some developmental contexts (14).

$D l x 1$ and $D l x 2$ were hypothesized to be important for ENS development over 20 years ago, but their role in the ENS was never carefully evaluated. $D l \times 2^{-1-}$ mice die as neonates with massive proximal bowel distention attributed to abnormal motility (10). Unsurprisingly, $D l x 1 / 2^{-/-}$mice have a similar phenotype due to a deletion encompassing $D l x 1, D l x 2$, and intergenic regions. In contrast, $D l x 1^{-1-}$ mice on some genetic 
backgrounds were reported to die by 1 month of age $(14,15)$, a time frame similar to mouse models with defined enteric neuropathies $(12,16)$. Consistent with the hypothesis that $D l x 1$ and $D l x 2$ mutations affect ENS function or development, both genes are expressed in developing ENS at ages when ENCDCs are migrating, proliferating, and differentiating into neurons and glia, including embryonic day 12.5 (E12.5) $(10,17)$, E14.5 (18, 19), E17.5 (20), and postnatal day 0 (P0) (18). Further supporting a role in ENS development, Dlx2 enhances expression of the transcription factor $Z f h x 1 b$ (also called SIP1 and Zeb2) in the CNS (21), and ZFHX1B mutations can cause Hirschsprung disease (a problem where distal bowel lacks ENS) $(22,23)$. Given compelling evidence implicating DLX1 and DLX2 in ENS development, we were surprised to find no studies thoroughly characterizing the ENS in mice lacking these proteins.

To evaluate DLX1 and DLX2's role in ENS development, we analyzed bowel structure and function in $D l \times 1 / 2^{-/-}$mice, which die at P0, and $D l x 1^{-/-}$mice, which survive to adulthood in our colony. We observed serious bowel function defects in $D l \times 1 / 2^{-/-}$mice at P0, including slower transit and absence of neurally mediated contractions. Given the critical role of DLX1 and DLX2 in CNS interneuron migration, we initially hypothesized that ENS precursor migration might also be defective in $D l \times 1 / 2^{-1-}$ mice, causing Hirschsprung-like disease (absent distal ENS) that explained dysfunctional bowel. To our surprise, ENCDC migration, neuronal and glial density, and ratios of neuronal subtypes were normal in $D l x 1 / 2^{-1-}$ mice. To define mechanisms causing defective bowel function, we performed RNA sequencing (RNA-seq) on E14.5 and P0 Dlx $1 / 2^{+/+}$and Dlx $1 / 2^{-/-}$ENCDCs. We identified dysregulation of many genes, including reduced expression of the neurotransmitter vasoactive intestinal peptide (Vip). We discovered fewer VIP-lineage neurons and reduced neuronal VIP expression in $D l \times 1 / 2^{-/-}$ENS, which may explain the functional defects in $D l \times 1 / 2^{--}$mice. To our knowledge, this study is the first linking $D l x$ genes to Vip expression.

\section{Results}

Dlx $1 / 2^{-1-}$ and Dlx $2^{-1-}$ mice die as neonates with massive abdominal distention due to intestinal gas accumulation. To evaluate the role of $D l x 1$ and $D l x 2$ in bowel development, we bred to generate $D l x 1^{-/-}, D l \times 2^{-/-}$, and $D l \times 1 / 2^{-1-}$ mice. The $D l \times 1 / 2^{-1-}$ mice appeared ill as neonates, lacked a milk spot, and died within 24 hours

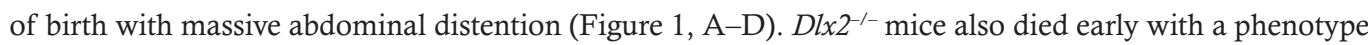
similar to that of $D l \times 1 / 2^{-/-}$mice, but survival was not tracked as closely; $D l \times 2^{-1-}$ mice were never recovered at weaning (P30). Gross anatomic analysis demonstrated marked accumulation of gas within the proximal SI (Figure 1, E and F). In contrast, $D l \times 1^{1^{--}}, D l \times 2^{+/-}, D l \times 1 / 2^{+/-}$, and wild-type (WT) mice appeared healthy as neonates. Furthermore, although $D l x 1^{-/-}$mice were previously reported to grow slowly and die by 1 month of age (12), we did not observe excess perinatal death of our $D l x 1^{-/-}$mice (Figure $\left.1 G\right)$ despite a slow growth trajectory (Figure 1, H and I). We note that our Dlx $1^{-1-}$ mice have CreERT2 inserted into the initiation codon, while Qiu et al. (12) deleted $D l x 1$ exons 2 and 3. Furthermore, our mice differed in strain background and mouse colony, which may account for survival differences. To test if $D l x 1^{-/-}$mice had poor bowel motility, which can cause slow growth, we gavage-fed 37-day-old $D l x 1^{-/-}$mice with fluorescein isothiocyanate-dextran (FITC-dextran) and evaluated transit through the bowel lumen. FITC-dextran is poorly absorbed across bowel epithelium, and transit of FITC-dextran through the bowel lumen is a well-established method to assess motility $(20,24)$. We observed no delays in SI transit (Figure 1, J and K). To assess colon motility, we inserted a glass bead into the distal colon of adult $D l x 1^{-/-}$and WT mice and timed bead expulsion. We observed a delay in distal colon bead expulsion consistent with dysmotility that did not impact survival (Figure 1L).

We noted that $D l \times 2^{-/-}$and $D l \times 1 / 2^{-/-}$mice have cleft palate, as previously reported $(10,12,25)$, and that this might cause poor feeding and air swallowing, but the marked accumulation of air in the proximal SI suggested that bowel dysmotility slows gas transit. We decided to focus analyses on $D l x 1 / 2^{-1-}$ mice that lack both DLX1 and DLX2 proteins because some functional redundancy has been reported for $D l x 1$ and $D l \times 2$ in the CNS.

Dlx $1 / 2^{-1-}$ mice have bowel dysmotility. To determine if bowel dysmotility at $\mathrm{P} 0$ contributes to bowel distension in $D l x 1 / 2^{--}$mice, we fed neonatal mice FITC-dextran and evaluated transit through the bowel lumen. To ensure conditions for testing were as similar as possible between genotypes, we delivered mice by Cesarean section at E19.5 (the usual day of delivery). Cesarean delivery prevented WT and Dlx $1 / 2^{+/-}$from feeding and permitted evaluation at a well-defined interval after birth. To delay spontaneous delivery but test bowel transit in full-term pups, mothers were treated with progesterone at E18.5. Between 1 to 3 hours after delivery, each pup received a small bolus of FITC-dextran delivered directly into the oral cavity. All mice swallowed FITC-dextran even when they had cleft palate. Three hours later, pups were euthanized and intraluminal FITC 

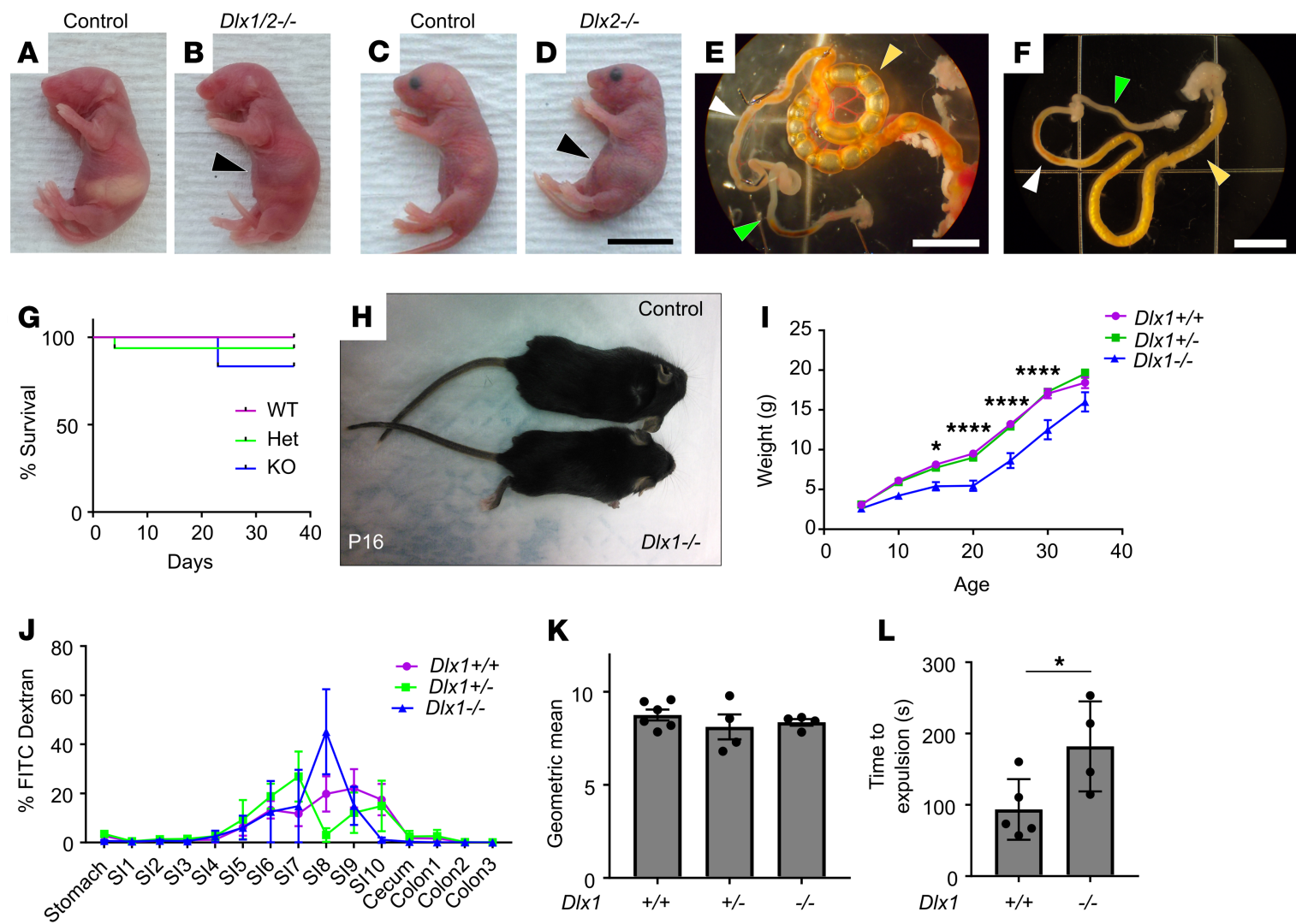

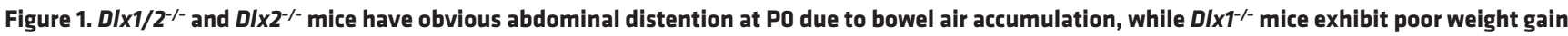
but normal small intestine (SI) transit. (A-D) $D I \times 1 / 2^{-/_{-}^{-}}$(B) and $D / \times 2^{-{ }^{--}}$(D) mice exhibited dramatic bowel distention (black arrowhead) compared to WT littermates (A and $\mathbf{C}$ ). Scale bar: $1 \mathrm{~cm}$. (E) Bowel of $D / x 2^{-1-}$ mouse viewed under dissecting microscope. Note air-filled proximal SI (yellow arrowhead) with absence of air in distal SI (white arrowhead) and colon (green arrowhead). Scale bar: $5 \mathrm{~mm}$. (F) Control bowel for comparison. Scale bar: $5 \mathrm{~mm}$. (G) Kaplan-Meier curve indicates most $D I x 1^{-/-}$mice survived past 1 month of age $(P=0.4665$; log-rank Mantel-Cox; $n=9[+/+], 16[+/-], 6[-/-])$. (H and I) $D / x 1^{-/-}$mice were small and weighed significantly less than their WT littermates at P15 $(P=0.0433), \mathrm{P} 20(P<0.0001), \mathrm{P} 25(P<0.0001)$, and P30 $(P<0.0001) ; n=9[+/+], 14[+/-]$, and 5 [-/-]; 2-way repeated-measures ANOVA. We include only mice that lived to P35. (J) Percentage FITC-dextran in distinct bowel regions 1 hour after oral FITC-dextran administration. SI1 to SI10 indicate sequential SI segments. (K) Weighted average of FITC bowel transit showed normal SI transit times in $D / x 1^{-/-}$mice $(P=0.5182$; 1-way ANOVA, $n=6[+/+], 4[+/-]$, and $4[-/-])$. (L) $D / x 1^{-/-}$mice had slight delays in expulsion of a glass bead from distal colon compared with WT controls $(P=0.0399$, Student's $t$ test, $n=5[+/+]$ and $n=4[-/-])$. ${ }^{*} P<0.05 ;{ }^{* * *} P<0.0001$.

fluorescence intensity was measured for defined bowel regions. In WT and $D l x 1 / 2^{+/-}$mice, most FITC-dextran reached mid-SI after 3 hours. In contrast, $D l \times 1 / 2^{-/-}$mutants retained most FITC-dextran in their stomach, with small amounts in the proximal SI (Figure 2A). A weighted average of fluorescence intensity (i.e., geometric mean) confirmed significant delays in bowel intraluminal transit for newborn $D l \times 1 / 2^{-/}$mutant mice (Figure 2B), suggesting abnormal SI motility.

Despite our attempts to control for the presence of bowel air, many $D l \times 1 / 2^{-1-}$ mice accumulated air in their bowel during the assay. Intriguingly, a subset of $D l \times 1 / 2^{+/-}$heterozygotes also spontaneously accumulated air in their GI tracts in the absence of cleft palate. Compared with these $D l x 1 / 2$ heterozygotes with intraluminal air, $D l \times 1 / 2^{-/-}$mice still had significantly slower FITC-dextran transit (Figure $2 \mathrm{C} ; P<0.05$ ), confirming poor gut motility in $D l \times 1 / 2^{-/-}$mutant mice.

One possibility was that delayed FITC-dextran transit in $D l \times 1 / 2^{-/-}$mice could reflect poor health of the $D l \times 1 / 2^{-1-}$ neonates (i.e., ileus as a result of systemic illness). In fact, $D l \times 1 / 2^{-1-}$ mice showed signs of poor health and $4 \mathrm{Dl} \times 1 / 2^{-/-}$pups died during the experiment. We excluded these animals and an additional 2 pups from our analysis due to lack of activity after the 3-hour study was complete. $D l \times 1 / 2^{-/-}$pups included in the experiment were more likely to be pale appearing and exhibited fewer movements in the first few hours of life (Supplemental Figure 1, A and B; supplemental material available online with this article; https://doi. org/10.1172/jci.insight.131494DS1). These concerns prompted us to perform an in vitro motility assay to complement our in vivo studies. We again treated dams with progesterone and delivered pups via Cesarean 
A
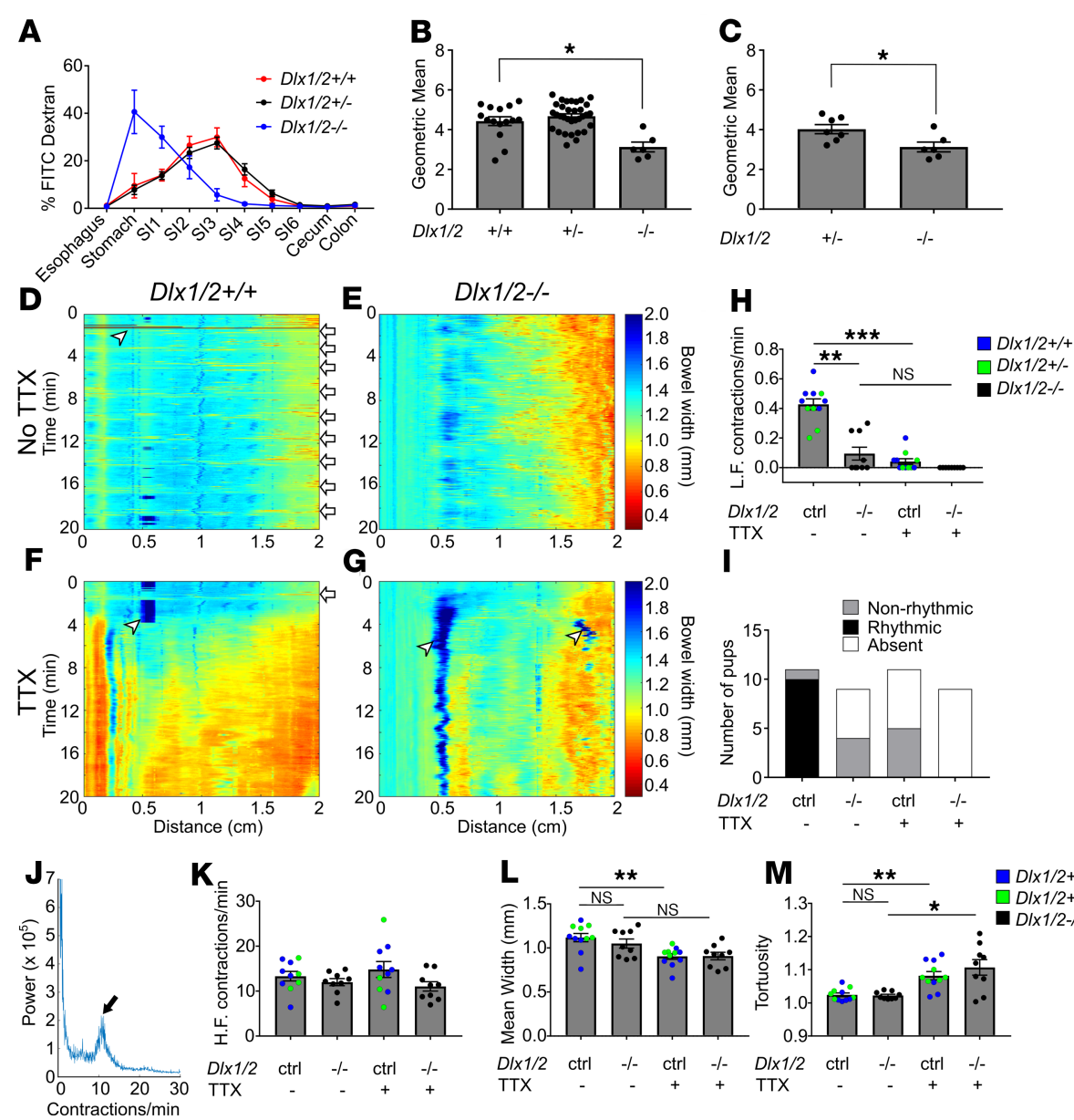

Figure 2. DIx1/2-/- mice have abnormal bowel motility. (A) Percentage FITC-dextran in distinct bowel regions 3 hours after oral FITC administration. SI1 to SI6 indicate sequential small intestine (SI) segments. (B) Weighted average of FITC bowel transit, represented by geometric mean, shows significant delays in transit for PO D/x1/2 $2^{-/-}$mice, but not for heterozygous DIx1/2+/- mice $(P=0.0188$; Kruskall-Wallis with Dunn's multiple-comparisons test; $n=15$ [+/+], 33 [+/-], $6[-/-])$. (C) A subset of heterozygotes spontaneously accumulated bowel air, but transit in $D / x 1 / 2^{-/-}$mutants was still slower than in D/x1/2+/- mice. ( $P=0.0228$; Student's $t$ test; $n=7[+/-], n=6[-/-])$. (D-G) Representative kymographs depicting bowel width (color axis) over time (y axis) at specific distances along the SI ( $x$ axis) in control and DIX1/2 $2^{-/-}$bowels in the absence ( $\mathbf{D}$ and $\left.\mathbf{E}\right)$ or presence (F and $\mathbf{G}$ ) of tetrodotoxin (TTX). White arrows indicate contraction complexes in control mice, but similar contraction complexes could not be identified in D/x1/2-/- bowel; white arrowheads indicate artifacts such as air bubbles on the surface of bowel. (H) $D / \times 1 / 2^{-/-}$mice had fewer low-frequency (L.F.) contraction complexes than controls $(P=0.0055$; Kruskal-Wallis with Dunn's multiple-comparisons test; $n=10$ [ctrl], $n=9[-/-])$. (I) Low-frequency contraction complexes generally occurred in rhythmic patterns in controls but not in mutants. (J) Representative Fourier plot from control mouse, with black arrow highlighting peak contraction frequency for TTX-insensitive contractions; note that contraction frequency for neurally mediated contractions is too low to be reliably identified on the Fourier graph. (K) High-frequency (H.F.) TTX-insensitive contractions occurred at normal rates in DIX1/2/-/- mice ( $P=0.181$; 1-way ANOVA; $n=10$ [ctrl], $n=9$ [-/-]). ( $\mathbf{L}$ and $\mathbf{M})$ In controls, average bowel width decreased significantly with addition of TTX (L; $P=0.0043$; 1-way ANOVA with Tukey's multiple-comparisons test), while tortuosity increased with TTX (M; $P=0.0087$; Kruskal-Wallis with Dunn's multiple-comparisons test). In mutants, bowel width did not significantly change with TTX addition ( $\mathbf{L} ; P=0.1423$ ), but tortuosity increased with $\operatorname{TTX}(\mathbf{M} ; P=0.0276)$. ${ }^{*} P<0.05 ;{ }^{* *} P<0.01 ;{ }^{* *} P<0.001$. Error bars represent SEM. NS, not significant.

section to control for food and air in the bowel. The bowel was carefully removed from neonates shortly after delivery and was placed in warmed, oxygenated Krebs-Ringers solution in a continuously perfusing organ bath. The SI was cannulated at the proximal end and perfused with oxygenated Krebs-Ringers using an oral pressure of $1.3 \mathrm{~cm} \mathrm{H}_{2} \mathrm{O}$ above the bowel lumen. Videos of bowel contractions were recorded in the presence or absence of tetrodotoxin (TTX) (see Supplemental Videos 1 and 2 for representative videos). Videos were converted to spatiotemporal maps or kymographs depicting bowel width (color axis) over time ( $y$ axis) for the first $2 \mathrm{~cm}$ of SI (Figure 2, D-G). In control bowel, we observed low-frequency ( 0.4/ 
minute) and high-frequency (6-18/minute) contractions, as has been previously described in neonatal mice (26). The low-frequency contraction complexes that typically occurred at regular intervals (i.e., rhythmically) in control mice were abrogated by TTX (Figure $2 \mathrm{H}$ ), indicating neuronal origin. The $D l x 1 / 2^{-/-}$mice had many fewer low-frequency contraction complexes than controls $(P=0.0055$; Figure $2 \mathrm{H})$ and none of the contraction complexes in Dlx $1 / 2^{-/-}$mouse SI occurred in a rhythmic pattern (Figure 2I). This analysis confirmed that neurally mediated motility patterns are abnormal in the SI of $D l \times 1 / 2^{-1-}$ mice.

Unlike low-frequency contractions, high-frequency contractions were present and appeared similar in control $\left(D l \times 1 / 2^{+/+}\right.$and $\left.D l \times 1 / 2^{+/-}\right)$and $D l \times 1 / 2^{-/-}$SI. These more frequent contractions are probably mediated by interstitial cells of Cajal or smooth muscle since they are TTX insensitive (26). Furthermore, Fourier transform analysis of the frequency of these faster contractions (Figure 2J) as described previously (26) showed no difference between control and $D l \times 1 / 2^{-/-}$mutant bowel (Figure $2 \mathrm{~K}$ ), consistent with a neuropathic origin of $D l \times 1 / 2^{-/-}$bowel dysmotility.

We next tested if the SI in $D l \times 1 / 2^{-/-}$mice was tonically contracted or dilated, problems that might suggest an imbalance in excitatory or inhibitory motor neuron signaling and could slow bowel intraluminal transit. Our analysis showed that $D l \times 1 / 2^{-/-}$bowel was neither more dilated nor more contracted than control bowel (Figure 2L). Baseline tortuosity (measured as length of pinned bowel divided by Euclidean distance from end to end in randomly selected video frames) was also no different in $D l \times 1 / 2^{-/-}$bowel (Figure 2M). Control bowel became more contracted and tortuous on exposure to TTX (Figure 2, L and M), suggesting that neural activity is necessary for baseline bowel relaxation in P0 SI. $D l \times 1 / 2^{-1-}$ bowel did not become significantly more contracted in the presence of TTX $(P=0.1423)$, but became more tortuous with TTX application $(P=0.0276)$, consistent with a distinct pattern of neuromodulation in $D l \times 1 / 2^{-/-}$mutant bowel (Figure 2, L and M). Collectively, these in vitro and in vivo data suggest that bowel motility is profoundly abnormal in $D l \times 1 / 2^{-1-}$ mice and that dysmotility is due to defects in neuronal activity.

Dlx 1 and Dlx2 are not necessary for bowel colonization by ENCDCs during fetal development. Because bowel motility was abnormal in $D l \times 1 / 2^{-1-}$ mice, we tested the hypothesis that DLX1 and DLX2 influence colonization of the bowel by ENCDCs during fetal development. We analyzed bowel at E12.5 when ENCDCs have normally colonized the entire SI and half of the colon. The extent of ENS precursor migration was assessed using an antibody against neuron-specific class III $\beta$-tubulin (TuJ1), which is expressed at high levels in enteric neurons, including at the migration wavefront $(27,28)$. By this criterion, E12.5 Dlx1/2-1- mice had normal colonization by ENCDCs (Figure 3, A-C). In contrast, $D l x 2^{-/-}$mice had a small reduction in bowel colonization by ENCDCs of dubious functional significance and marginal statistical significance $(66.5 \% \pm 1.5 \%$ colonized $[+/+]$ vs. $47.4 \% \pm 5.2 \%$ colonized $[-/-] ; P=0.0499$; Figure 3, E-G), perhaps reflecting a relatively protective CD1 strain background for $D l x 1 / 2$ compared with the aganglionosis-promoting C57BL/6J strain background (29) for $D l x 2^{-/-}$mice. Because some gene defects are only apparent with a sensitized genetic background (30), we next evaluated bowel colonization by $D l \times 1 / 2^{-/-}$ENCDCs in the context of Ret heterozygosity. Dlx $1 / 2^{-/-}$Ret $^{+/-}$mice on a mixed CD1 $\times$ C57BL6/J background had apparently normal colonization of the bowel by ENCDCs (Figure 3, I-K). Colon length was also normal in all mutant mice (Figure 3, D, H, and L). These observations suggest that bowel dysmotility in $D l x 1 / 2^{-/-}$mice is not due to abnormal bowel colonization by ENCDCs.

To determine if $\mathrm{Dl} \times 1 / 2^{--}$mice have major ENS structural defects causing dysmotility, we dissected mutant mice just after birth (P0) and stained bowel whole mounts with antibodies against the neuronal RNA-binding protein HuC/D (ANNA-1), TuJ1, SOX10 (which is present in ENS precursors and mature glia), and the glia-specific marker $S 100 \beta$. There were no obvious differences in ENS organization or staining patterns between $D l \times 1 / 2^{+/+}$and $D l \times 1 / 2^{-/-}$mice. TuJ1 staining showed normal organization of nerve fibers. Quantitative analysis of $\mathrm{HuC} / \mathrm{D}$ staining demonstrated normal neuronal density in proximal SI, distal SI, and colon of P0 Dlx1/2-/- mice (Figure 4, A-O). For SI, we assessed neuronal density in both myenteric and submucosal plexus, but due to difficulty separating muscle from submucosa in neonatal mice, we were only able to assess total neuronal density (myenteric plexus + submucosal plexus) in colon. P0 Dlx1/2-/ mice also had normal SOX $10^{+}$cell density and SOX $10^{+} S 100 \beta^{+}$glial density in bowel regions examined (Figure 4, P-W).

Because neuron death occurs in subsets of CNS neurons in $D l x 1^{-/-}$mice postnatally (15), and because we observed slightly delayed colon motility, we assessed neuronal numbers in 10 -week-old $D l x 1^{-1-}$ mice, which lack cleft palate and survive to adulthood. We found no differences in neuronal density in proximal SI, distal SI, mid-colon, or distal colon in $D l x 1^{-/-}$adults in either myenteric or submucosal plexus (Figure 5, A-X). We also found no difference in SOX10 ${ }^{+}$glia and ENS precursors in distal SI of mature $D l x 1^{-/-}$mice (Figure $5, \mathrm{Y}-\mathrm{AA}$ ). 

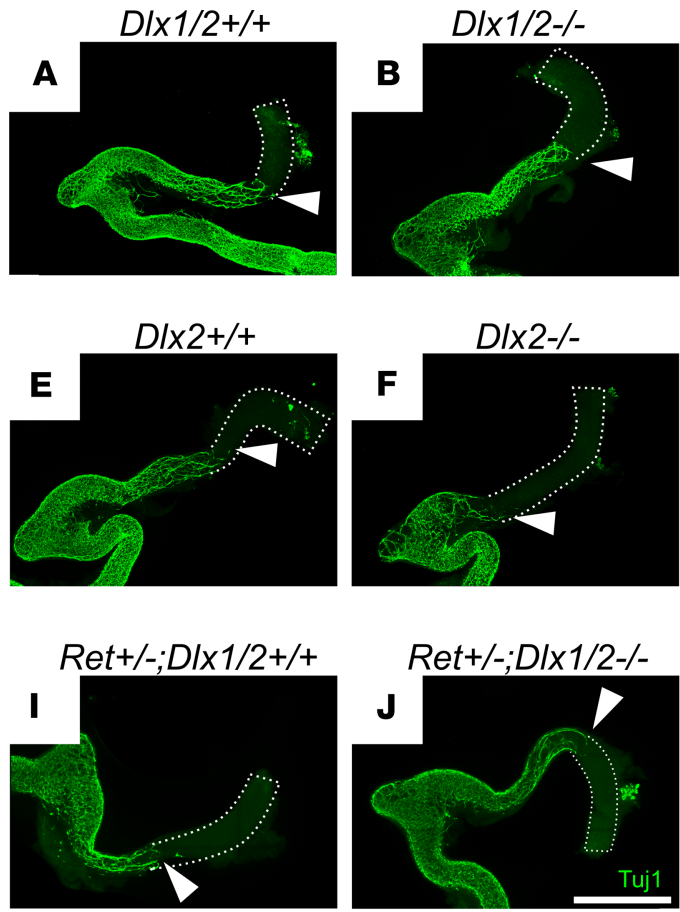
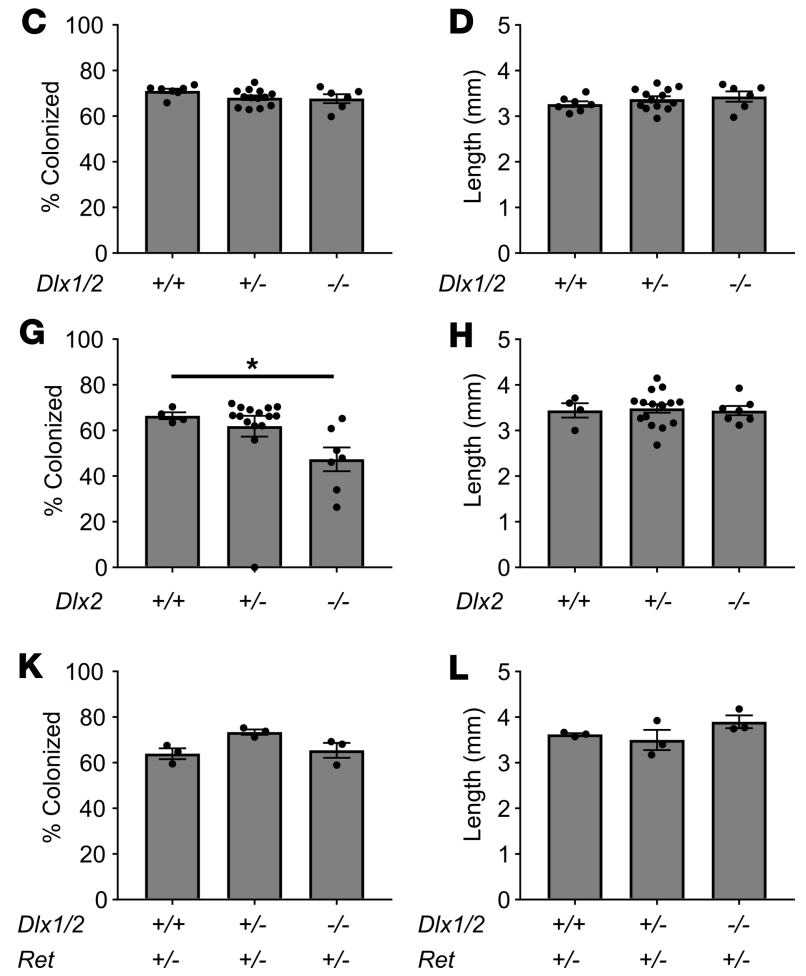

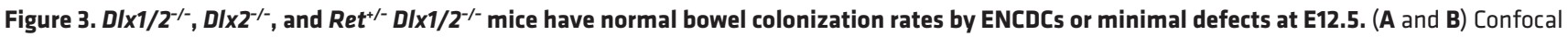
Z-stacks of WT (A) and DIx1/2 $2^{-/-}$(B) E12.5 bowel stained with Tul1 antibody that labels early and mature neurons (green). Maximum intensity projections are shown. White triangles mark distal-most TuJ1+ neuron processes. Scale bar: $1 \mathrm{~mm}$. (C and D) Percentage hindgut colonized relative to total colon length (C; 1-way ANOVA, $n=7[+/+], n=13[+/-], n=6[-/-]$ ) and colon length (D; 1 -way ANOVA, $n=7[+/+], n=13[+/-], n=6[-/-])$ were normal in D/x1/2-/-

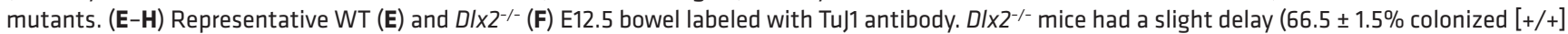
vs. $47.4 \pm 5.2 \%$ colonized [-/-]) in ENS migration of unknown functional significance (G; $P=0.0499$; Kruskal-Wallis test with Dunn's multiple-comparisons

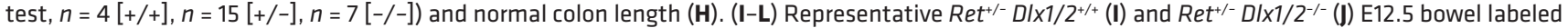
with TuJ1 antibody. DIX1/2-/- mice on a Ret-heterozygous background had normal extent of bowel colonization (I; 1 -way ANOVA, $n=3$ per genotype) and normal colon length (L; 1 -way ANOVA, $n=3$ per genotype). Scale bar: $1 \mathrm{~cm}$. ${ }^{*} P<0.05$.

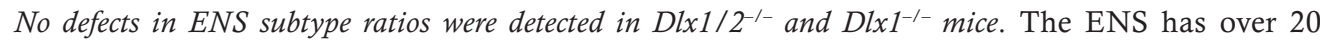
neuronal subtypes that perform distinct functions (31). Because total neuron numbers are normal in Dlx $1 / 2^{-1-}$ mice, but bowel motility is abnormal, we hypothesized that DLX1 and DLX2 might be required for differentiation or survival of 1 or more neuronal subtypes, leading to dysmotility in Dlx $1 / 2^{-/-}$mice. To test this hypothesis, we stained P0 bowel with antibodies that distinguish many types of enteric neuron. We observed no differences in ratios of neurons expressing myenteric plexus neurotransmitter markers nitric oxide synthase (NOS), calretinin, $\gamma$-aminobutyric acid (GABA), or VIP (Figure 6, A-E, G-K, and M-Q) or the submucosal plexus neurotransmitter VIP (Figure 6, F, $\mathrm{L}$, and R). Because we lacked reliable antibodies for choline acetyl-transferase (ChAT), we bred and analyzed Dlx1/2 ChAT-EGFP-L10a mice but found no difference in $\mathrm{EGFP}^{+}$neuron number (Figure 6, A, G, and M, and Supplemental Figure 2, A-N). One technical issue is that the fluorescence intensity of immunohistochemical signals for certain neurotransmitters at P0 (substance P, tyrosine hydroxylase) was quite low (Supplemental Figure 3, A-D). We suspect this reflects incomplete differentiation of these subtypes at P0, because immunostaining in adult mouse ENS was excellent for all antibodies used (Supplemental Figure 3, E-J). Despite substantial effort, we were unable to identify neuronal populations that were dramatically altered at an anatomic level to explain the severe intestinal dysmotility in neonatal $D l \times 1 / 2^{-/-}$mice.

In $D l x 1^{-1-}$ mice, we evaluated ENS in adult bowel because the somatosensory cortex of the CNS has normal neuronal numbers at birth, but postnatal apoptosis occurs in several cortical neuron subtypes, including $\mathrm{GAD}^{+} 7^{+}$(which catalyzes GABA synthesis from glutamate), $\mathrm{NOS}^{+}$, somatostatin ${ }^{+}$, and calreti$\operatorname{nin}^{+}$neurons (15). Taking advantage of postnatal survival in $D l x 1^{-1-}$ mice, we determined if similar age-dependent neuronal subtype loss occurs in the $D l x 1^{1^{--}}$ENS by staining adult $D l x 1^{-/-}$distal SI with antibodies marking 

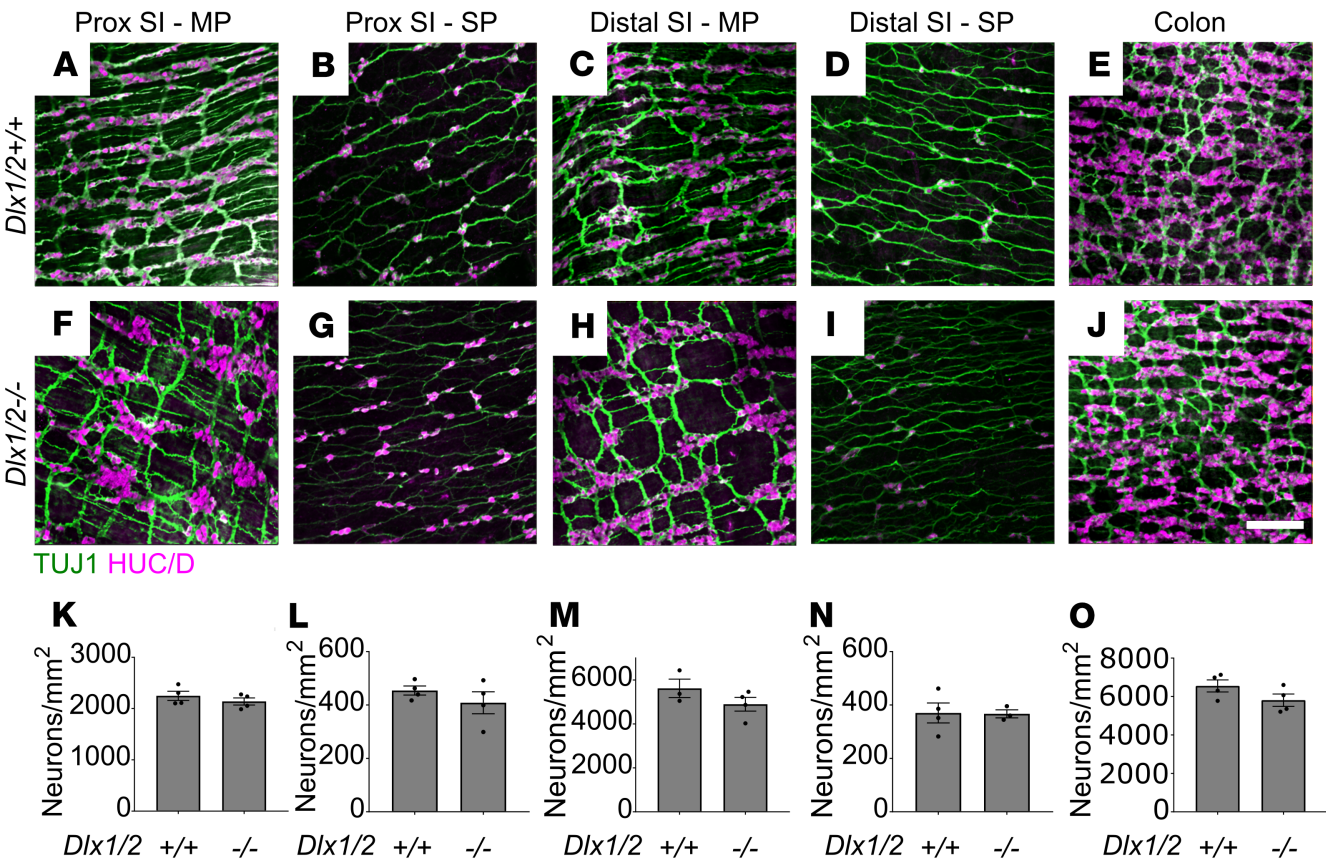

Proximal SI
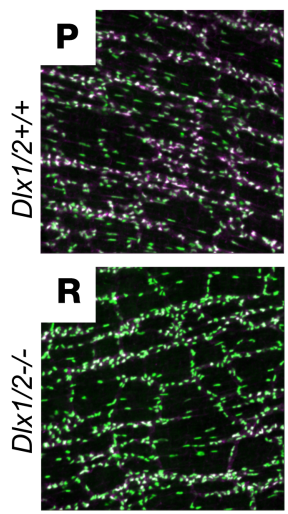

SOX10 S100ß
Distal SI

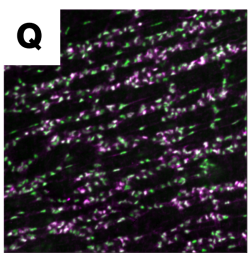

$\mathbf{s}$

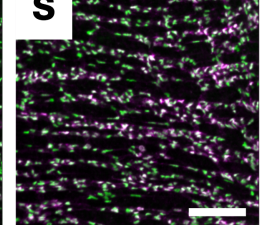

Proximal SI
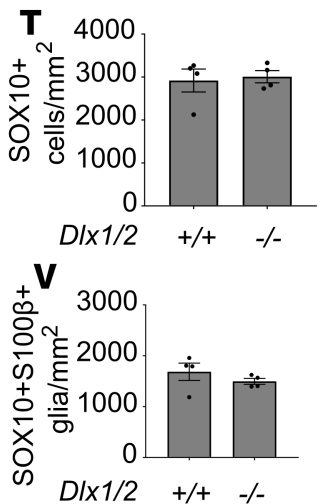

Distal SI
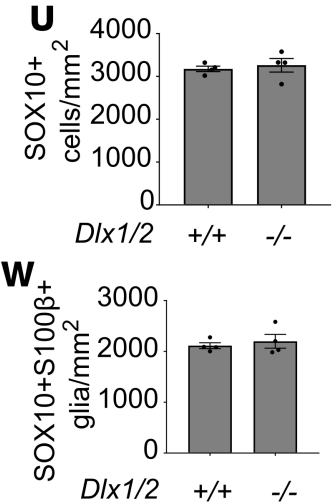

Figure 4. DIX1/2-/- mice have normal density of neurons, SOX10+ cells, and glia at PO. (A-J) Representative images of $D / \times 1 / 2^{+/+}$and $D / \times 1 / 2^{-/-}$bowel immunostained for HuC/D (magenta) and TuJ1 (green). MP, myenteric plexus; SP, submucosal plexus. (K-0) Quantification of neurons in A-J shows no difference between neuronal number in control or mutant mice (Student's $t$ test; $n=3-4$ per condition). (P-S) Representative images of $D / \times 1 / 2^{+/+}$and $D / \times 1 / 2^{-/-}$bowel immunostained for $\mathrm{S} 100 \beta$ (magenta) and S0X10 (green). (T-W) DIX1/2-/- mice had normal numbers of S0X10 cells at PO (Student's $t$ test; $n=3-4$ per condition; $\mathbf{T}$ and $\mathbf{U}$ ) and normal numbers of S0X $10^{+} S 100 \beta^{+}$glia at P0 (Student's $t$ test; $n=3-4$ per condition; $\mathbf{V}$ and $\mathbf{W})$. Scale bars: $100 \mu \mathrm{m}$.

NOS, GABA, somatostatin, and VIP. Neuronal subtype ratios were similar in WT and $D l x 1^{-/-}$mice (Figure $6, \mathrm{~S}-\mathrm{DD})$, suggesting that postnatal enteric neuron loss is not occurring.

Vip is dysregulated in E14.5 and PO Dlx1/2-/- ENCDCs. Because our detailed anatomic analyses failed to reveal obvious structural problems of the ENS of $D l \times 1 / 2^{-1-}$ mice, we next hypothesized that dysmotility in $D l \times 1 / 2^{-/-}$mice is due to altered gene expression not discernable at the level of ENS anatomy. We tested this hypothesis by RNA-seq of pooled ENCDCs isolated from E14.5 and P0 bowel using fluorescence-activated cell sorting (FACS). We chose these ages for theoretical and technical reasons. E14.5 was selected because $D l x 1$ and $D l \times 2$ are expressed in murine ENS by E12.5 (10, 17), and we wanted to evaluate early effects of mutations, rather than secondary effects that occur as development proceeds. Furthermore, at E14.5 there are many ENCDCs for analysis, and neuronal subtype markers are starting to appear as enteric neural networks are established. We also analyzed P0 ENS to match our 


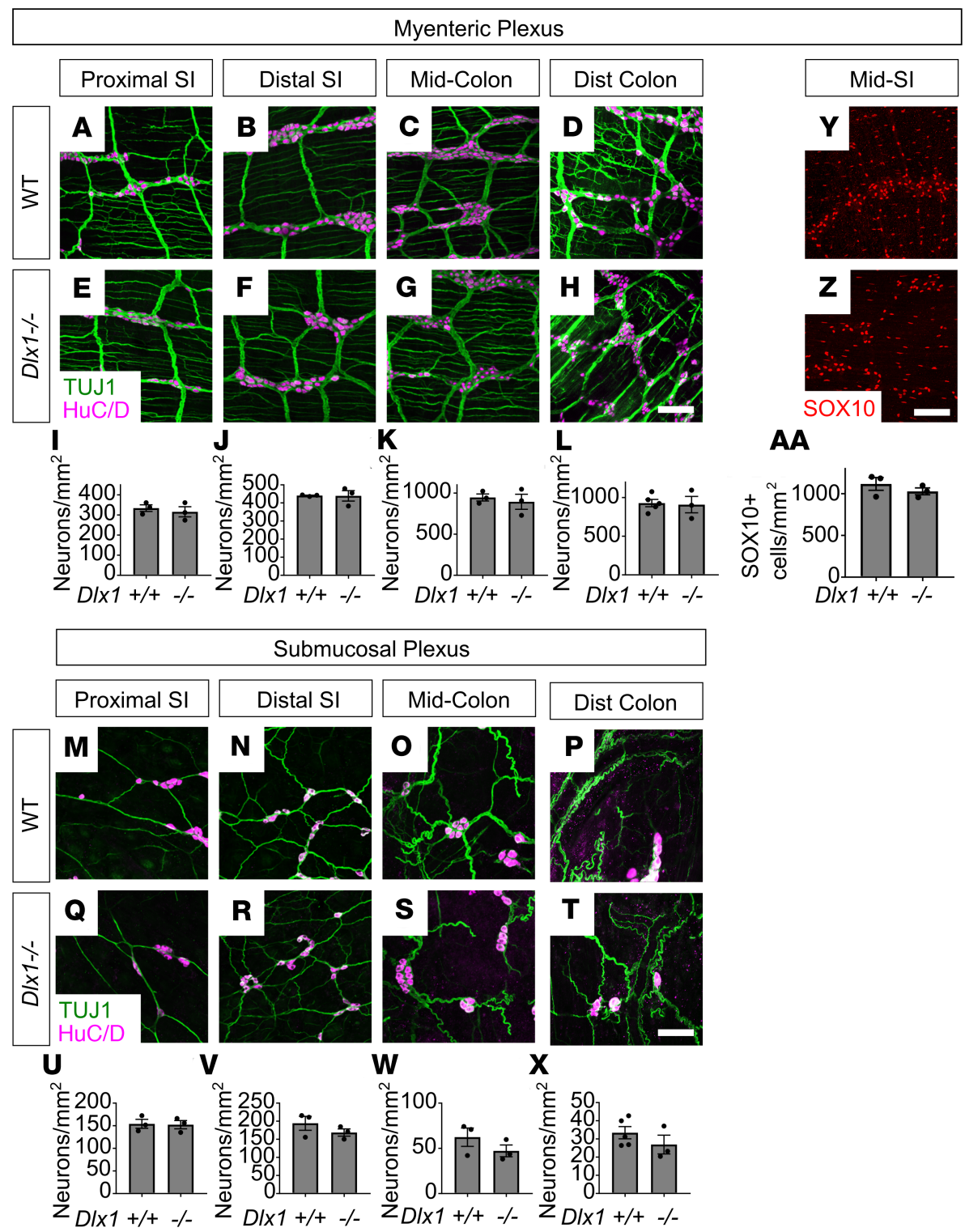

Figure 5. DIx1-/- adults have normal neuronal and glial numbers. (A-X) Representative images and quantifications of adult $D / \times 1^{-/-}$mouse proximal small intestine (SI), distal SI, mid-colon, and distal colon immunostained using HuC/D (magenta) and TuJ1 (green) antibodies. Myenteric plexus (A-H) and submucosal plexus (M-T) are shown. Neuronal

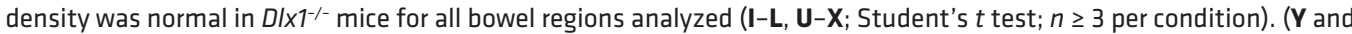
Z) Adult $D I \times 1^{-1-}$ distal $S I$ was immunostained using an antibody against SOX10, which exclusively labels glia in adults (red). (AA) Quantitative analysis of glial density shows no difference in glial cell density in distal SI of $D / x 1^{-1-}$ mice or WT mice (Student's $t$ test; $n=3$ per condition). Scale bars: $100 \mu \mathrm{m}$.

functional experiments performed using neonatal bowel. For our E14.5 analysis, we bred Dlx1/2 mice to an Ednrb-L10A-GFP reporter line that expresses a fluorescent protein in ENCDCs at E14.5 (32) and

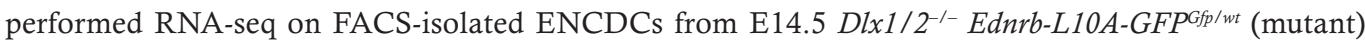
and Dlx1/2+/+Ednrb-L10A-GFP Gfp/wt (WT) SI. To analyze P0 bowel, we bred Dlx1/2 mice to a Wnt1-Cre R26R-Tdtomato line and performed RNA-seq on FACS-isolated ENS from P0 Dlx1/2-/ Wnt1-Cre ${ }^{\text {ere }}$ we R26R-Tdtomato ${ }^{+}$(mutant) and Dlx1/2 $2^{+/+}$Wnt1-Cre $e^{\text {cre } / \text { wt }} R 26 R$-Tdtomato ${ }^{+}$(WT) SI.

We identified 22 dysregulated genes in $D l \times 1 / 2^{-/-}$E14.5 ENCDCs using $q$ less than 0.1 as a statistical threshold after filtering out genes with low expression (average expression $<1$ ) and fold change $\left(\mid \log _{2}\right.$ fold change $\mid<1$; Figure 7, A and B). Given that DLX2 regulates Zfhx1b in the CNS, we had hypothesized that $Z f h x 1 b$ might be dysregulated in the $D l x 1 / 2^{--}$ENS, but we found no evidence of altered $Z f h x 1 b$ levels. Remarkably, however, we observed approximately $84 \%$ reduction in mRNA levels for the ENS 


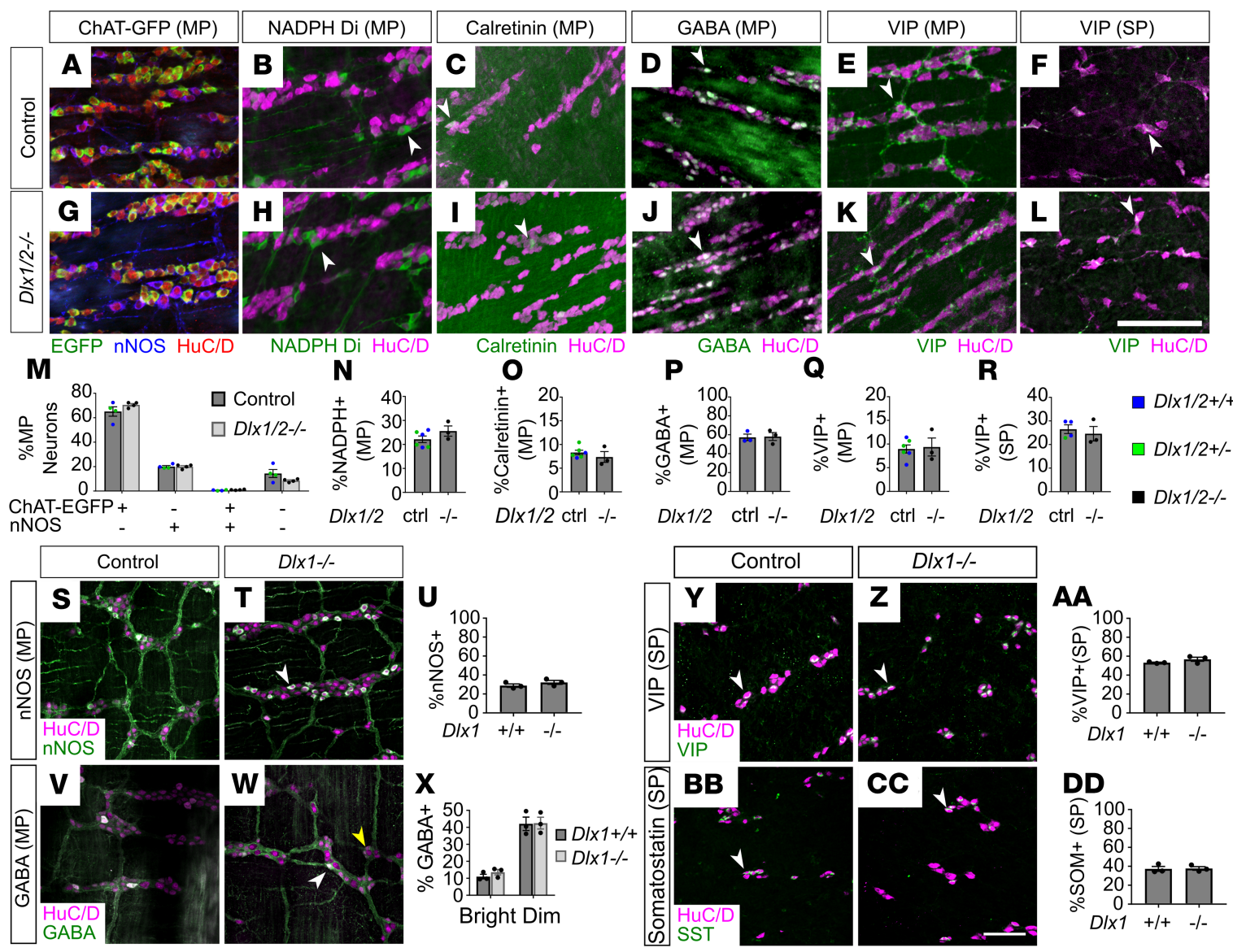

Figure 6. DIx1/2-/- PO and DIX1-/- adult mice have normal ratios of neuronal subtypes. (A-L) Representative images of HuC/D (red), nNOS (blue), and ChAT-EGFP (green; A and G), HuC/D (magenta) and NADPH-diaphorase (green; B and H), HuC/D (magenta) and calretinin (green; C and I), HuC/D (magenta) and GABA (green; $\mathbf{D}$ and J), HuC/D (magenta) and VIP (green; E, F, K, and L) immunostaining in PO control and PO D/x1/2-1- bowel. SP, submucosal plexus; MP, myenteric plexus. (M-R) We observed no difference in ratios of myenteric ChAT-EGFP or nNOS+ $(\mathbf{M})$, myenteric NADPH ${ }^{+}(\mathbf{N}), \mathrm{calretinin}^{+}(\mathbf{0}), \mathrm{CABA}^{+}(\mathbf{P}), \mathrm{VIP}^{+}$

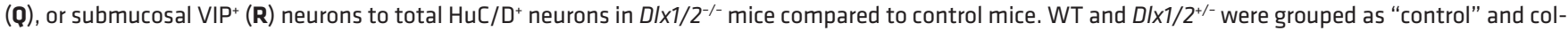
or coded $D I \times 1 / 2^{+/+}$(blue), $D I \times 1 / 2^{+/-}$(green) (Student's $t$ test, $n=3-6$ per condition). (S, T, V, W, Y, Z, BB, and CC) Representative images of HuC/D (magenta) and nNOS (green; $\mathbf{S}$ and T), HuC/D (magenta) and GABA (green; $\mathbf{V}$ and $\mathbf{W}$ ), HuC/D (magenta) and VIP (green; $\mathbf{Y}$ and $\mathbf{Z}$ ), and HuC/D (magenta) and somatosta-

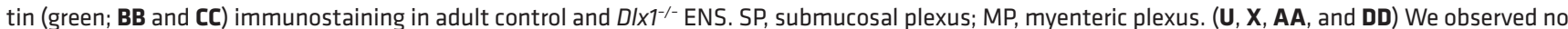

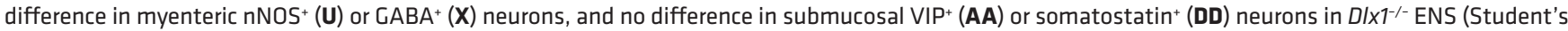
$t$ test, $n=3$ per condition). Arrowheads indicate examples of neurons that were counted. For GABAergic neurons in $D / x 1^{-/-}$adults, white arrowheads indicate brightly positive GABAergic neurons, while yellow arrowheads indicate dimly positive GABAergic neurons. Scale bars: $100 \mu \mathrm{m}$.

neurotransmitter Vip and a 3-fold increase in abundance of mRNA for the neurotransmitter proenkephalin (Penk) in SI ENCDCs from $D l x 1 / 2^{-1-}$ mice compared with WT animals. We also observed changes in receptors (Lifr), extracellular matrix genes (Mmp2, Col3a1), cytoskeletal regulators (Dnm3, Capn6, Fmn1), neurite growth factors (Ptn), and glia-associated genes (Plp1, Lrp1b) (Figure 7B). Because neurotransmitter dysregulation might most directly explain bowel dysmotility in $D l \times 1 / 2^{-/-}$mice, we attempted to validate altered levels of Vip and Penk mRNA using quantitative real-time polymerase chain reaction (qRT-PCR) on independently isolated E14.5 mutant and control samples (Figure 7, C and D; see Supplemental Figure 4 for standard curves). qRT-PCR analyses showed 3-fold reduction in Vip mRNA in E14.5 Dlx $1 / 2^{-/-}$ENCDCs compared with WT $(1.0 \pm 0.11[+/+]$ vs. $0.36 \pm 0.10[-/-], P=0.004)$ and no statistical difference in Penk mRNA $(1.0 \pm 0.02[+/+]$ vs. $1.34 \pm 0.17[-/-], P=0.063)$. Although we identified fewer dysregulated genes at P0, our RNA-seq data suggest Vip continues to be dysregulated in Dlx1/2-- neonatal ENS (Figure 7, E and F).

To further explore the VIP-expressing enteric neuronal subpopulations, we bred to generate Dlx1/2 Vip-IRES-Cre R26R-TdTomato mice, which produce TdTomato in cells expressing Vip. Although we had already counted VIP-immunoreactive enteric neurons, VIP is primarily in neurites, making cell counting suboptimal. We discovered that $\mathrm{TdTomato}^{+}$neurons in the mid-SI are significantly reduced in absolute 
A

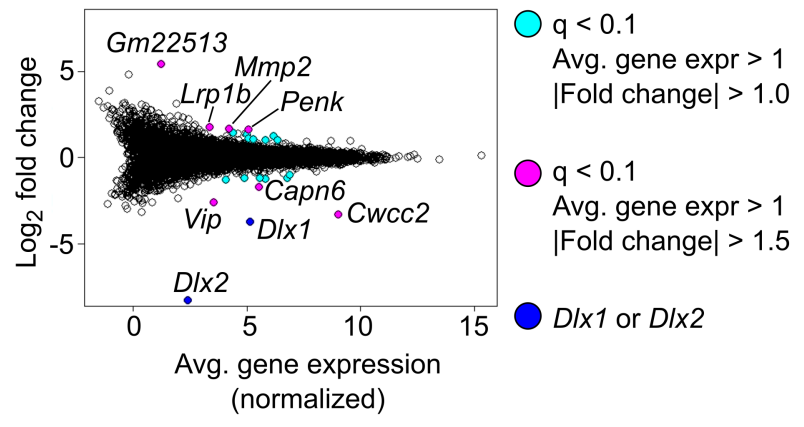

C

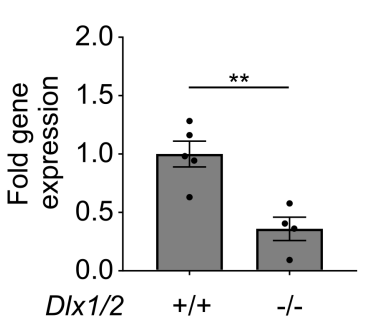

D Penk

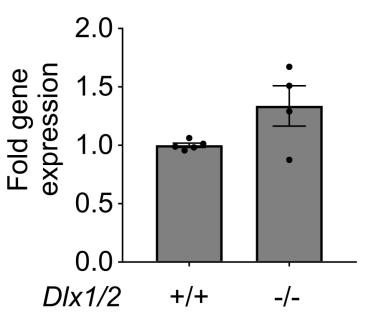

$\mathbf{E}$

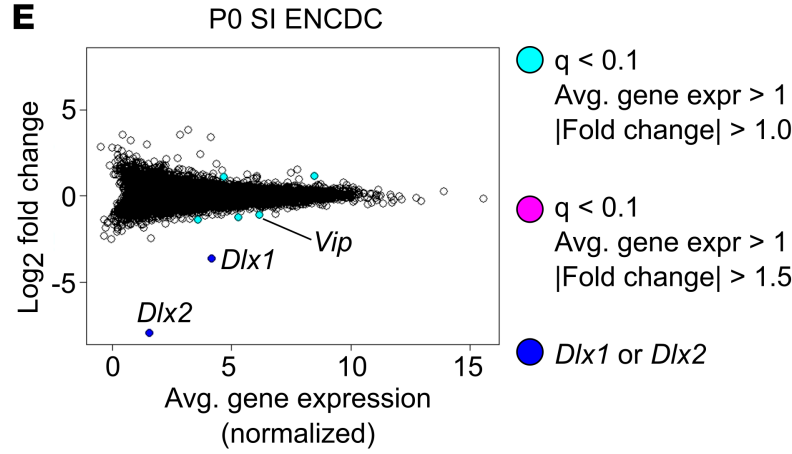

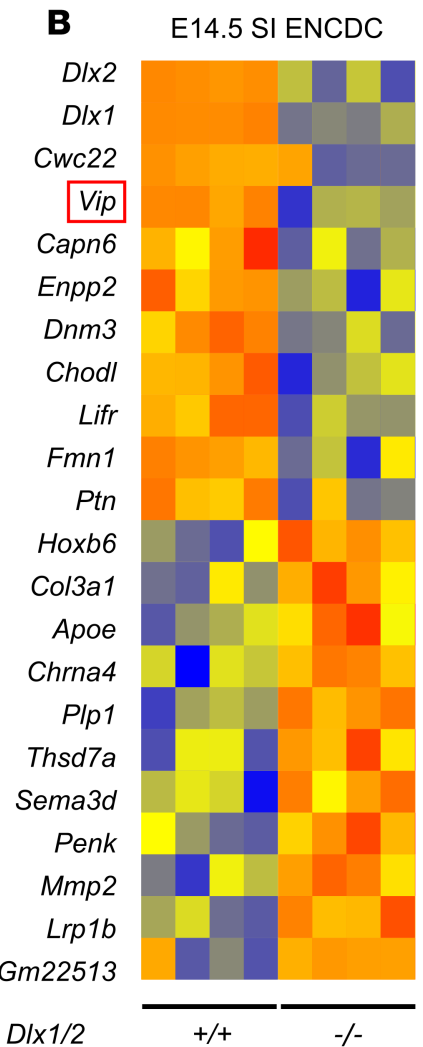

\begin{tabular}{cc} 
q-value & Log FC \\
0.0001 & -8.30 \\
0.0000 & -3.76 \\
0.0104 & -3.33 \\
0.0008 & -2.60 \\
0.0289 & -1.72 \\
0.0608 & -1.30 \\
0.0013 & -1.26 \\
0.0228 & -1.22 \\
0.0007 & -1.22 \\
0.0139 & -1.21 \\
0.0246 & -1.03 \\
0.0145 & 1.01 \\
0.0300 & 1.02 \\
0.0986 & 1.06 \\
0.0575 & 1.09 \\
0.0005 & 1.24 \\
0.0571 & 1.31 \\
0.0794 & 1.41 \\
0.0121 & 1.62 \\
0.0246 & 1.65 \\
0.0367 & 1.77 \\
0.0300 & 5.40 \\
\hline & \\
\hline & \\
\hline & \\
\hline Row Z-Score & \\
\hline & \\
\hline
\end{tabular}

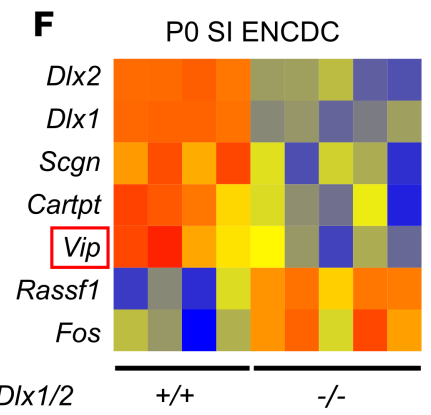

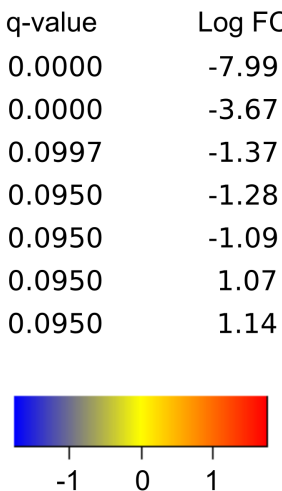

Row Z-Score

Figure 7. Vip levels are reduced in the developing ENS of DIx1/2-/- mice at E14.5 and PO. (A) Bland-Altman plot of differentially expressed genes in DIX1/2-- versus WT E14.5 FACS-isolated small intestine (SI) ENCDCs after RNA-seq. (B) Heatmap of differentially expressed genes shows 20 dysregulated genes in $D / x 1 / 2^{-/-}$ENS at E14.5, in addition to $D / x 1$ and $D / x 2$, which were substantially decreased in mutant mice. (C and D) Quantitative RT-PCR was performed for Vip (C) and Penk (D) mRNA on independent samples to validate expression patterns for these neurotransmitters in E14.5 mouse ENS (Vip, $P=0.0042$, Student's $t$ test, $n=5[+/+], n=4[-/-]$; Penk, $P=0.0634$, Student's $t$ test, $n=5(+/+), n=4[-/-])$. (E) Bland-Altman plot of differentially expressed genes in $D / x 1 / 2^{-/-}$versus WT PO FACS-isolated ENCDCs after RNA-seq. (F) Heatmap of differentially expressed genes shows 5 dysregulated genes in $D / x 1 / 2^{-1-}$ ENS at $P 0$, in addition to $D / \times 1$ and $D / x 2 .{ }^{* *} P<0.001$.

cell density (18.4\%) and relative to total neuron number (13.2\%) in myenteric plexus of Dlx1/2 Vip-IRES Cre R26R-TdTomato mice (Figure 8, A-D). Furthermore, VIP reporter (TdTomato ${ }^{+}$) neuronal density was dramatically reduced in absolute (51.5\%) and relative terms (41.0\%) in the submucosal plexus of $D l \times 1 / 2$ Vip-IRES-Cre R26R-TdTomato mice (Figure 8, F-I). As before, we saw no difference in total neuronal density (Figure 8, E and J). We also confirmed that most TdTomato ${ }^{+}$neurons appeared to express VIP protein at P0 (Supplemental Figure 5, A-C). 

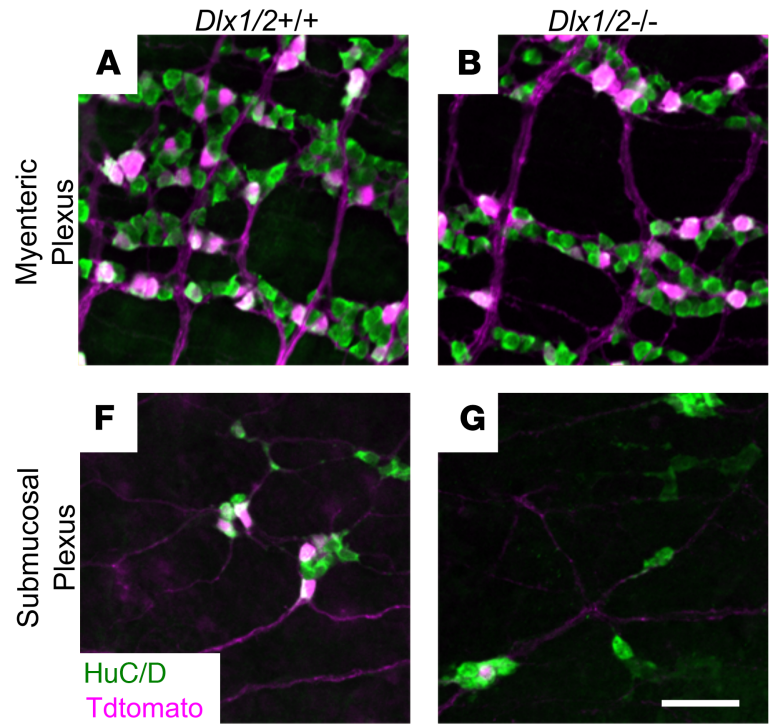
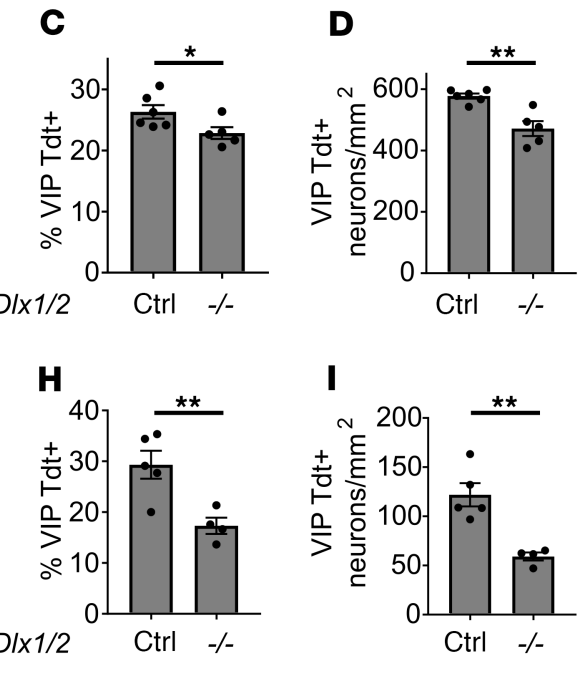

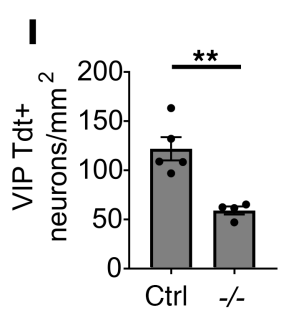

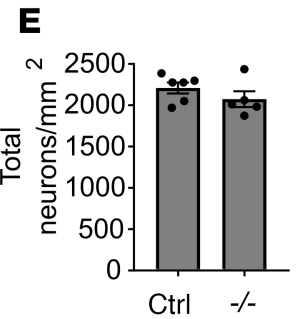

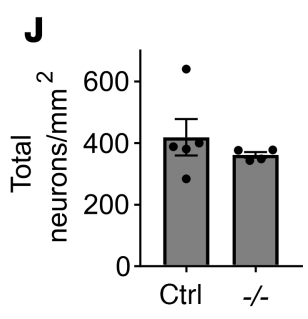

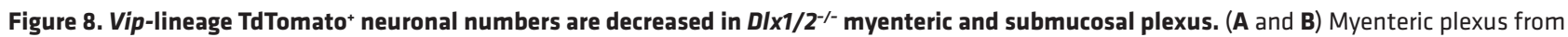
DIx1/2 //(+ or -) VIP-IRES-Cre ${ }^{\text {cre/wt }}$ R26R-TdTomato ${ }^{+}$control (A) and DIx1/2 ${ }^{-/-}$VIP-IRES-Cre ${ }^{\text {Cre/wt }}$ R26R-TdTomato mouse (B) stained with ANNA-1 antibody labeling HuC/D (green) reveals fewer TdTomato ${ }^{+}$cells in mice lacking DIx1/2. (C and D) Quantification of $\mathbf{A}$ and $\mathbf{B}$ shows a reduced proportion of TdTomato neurons in $D I x 1 / 2^{-/-}$myenteric plexus $(\mathbf{C} ; P=0.047, n=6$ [ctrl],$n=5[-/-])$ and reduced overall TdTomato neuronal density $(\mathbf{D} ; P=0.0017, n=6$ [ctrl], $n$ = $5[-/-]$ ). (E) Total MP neuronal density was unchanged in DIx1/2 mutant mice (E; $P=0.26, n=6[\mathrm{ctrl}], n=5[-/-])$. (F and G) DIx $1 / 2^{-/-}$VIP-IRES-Cre Cre/wt R26R-TdTomato mutants also have fewer TdTomato (red) HuC/D+cells in their submucosal plexus. (H and I) Quantification of F and G showing decreased proportion of TdTomato neurons relative to total $(\mathbf{H} ; P=0.0098, n=5$ [ctrl], $n=4[-/-])$ and decreased TdTomato ${ }^{+}$neuronal density $(\mathbf{I} ; P=0.0027, n=5$ [ctrl], $n=4[-/-])$. (J) Total submucosal plexus neuronal density was unchanged in $D / x 1 / 2^{-/-}$mice. Scale bar: $100 \mu \mathrm{m}$. ${ }^{*} P<0.05,{ }^{*} P<0.001$.

Dlx1/2-1- mice on a mixed background have fewer circular smooth muscle-projecting neurites. To determine if VIPergic neurites differed in mutants, we immunostained Dlx1/2 Vip-IRES-Cre R26R-TdTomato SI with an antibody against neurites (TuJ1). We first examined nerve fibers within the myenteric plexus. All large nerve fiber bundles were $\mathrm{TdTomato}^{+}$(Figure 9, A-H). We found no difference in nerve fiber bundle diameter between $D l \times 1 / 2^{-1-}$ mice and controls (Figure 9 , I and J).

To determine if the relative density of $\mathrm{VIP}^{+}$neurites projecting into circular smooth muscle (CSM) is altered in $D l \times 1 / 2^{-/-}$mice, we quantified neurite density by drawing a line perpendicular to the axis of CSM neurite projections and counting thin fibers crossing that line. Total density of CSM TuJ1+ neurites was significantly reduced in $D l x 1 / 2^{-1-}$ mice $(22.3 \%$ reduction, $P=0.0077$; Figure $9, \mathrm{~K}-\mathrm{Q})$. However, the ratio of $\mathrm{TdTomato}^{+}$neurites to total was not reduced (Figure 9R). TdTomato ${ }^{+}$neurite density was reduced but this reduction did not reach our threshold for statistical significance (Figure 9S). To determine if the altered neurite density was consistent across strain backgrounds, we quantified density of proximal SI CSM-projecting TuJ $1^{+}$neurites in $D l \times 1 / 2^{-/-}$mice on a pure CD1 background, but we found no difference in neurite density between mutants and controls $(P=0.3193$; Figure 9T). These data suggest $D l \times 1 / 2$ may affect targeting or growth of some neurites in CSM.

To further investigate how $D l x 1$ and $D l x 2$ affect neurite growth, we cultured ENCDCs from unselected E12.5 bowel in the presence of glial cell line-derived neurotrophic factor (GDNF) and labeled neurites using TuJ1. For this experiment, we used Dlx1/2 ChAT-EGFP-L10a mice on a mixed CD1 $\times$ C57BL/ 6 background, because we initially hoped to use EGFP to distinguish $\mathrm{ChAT}^{+}$and $\mathrm{ChAT}^{-}$neurons in culture; however, EGFP was not expressed at this young age. Cultured neurons exhibited highly variable morphology (Supplemental Figure 6, A and B). Quantification of total neurite length per cell body revealed no obvious differences between $D l x 1 / 2^{-1-}$ and WT neurons (Supplemental Figure $6 \mathrm{C} ;>400$ cells traced), and histograms of total neurite length for individual traced cells were similar for mutant and WT neurons (Supplemental Figure 6, D and E).

Dlx1/2-1- mice have reduced VIP in myenteric plexus nerve fibers and soma. To determine if VIP is reduced in myenteric nerve fibers, we immunostained P0 mouse SI with antibodies against TuJ1, HuC/D, and VIP and imaged at high power. Using CellProfiler image analysis software, we created a mask defined by TuJ1, but not HuC/D (i.e., myenteric plexus nerve fibers, with cell bodies excluded) and computed average VIP intensity within the masked region (Figure 10, A-K). Average VIP intensity within myenteric plexus nerve 

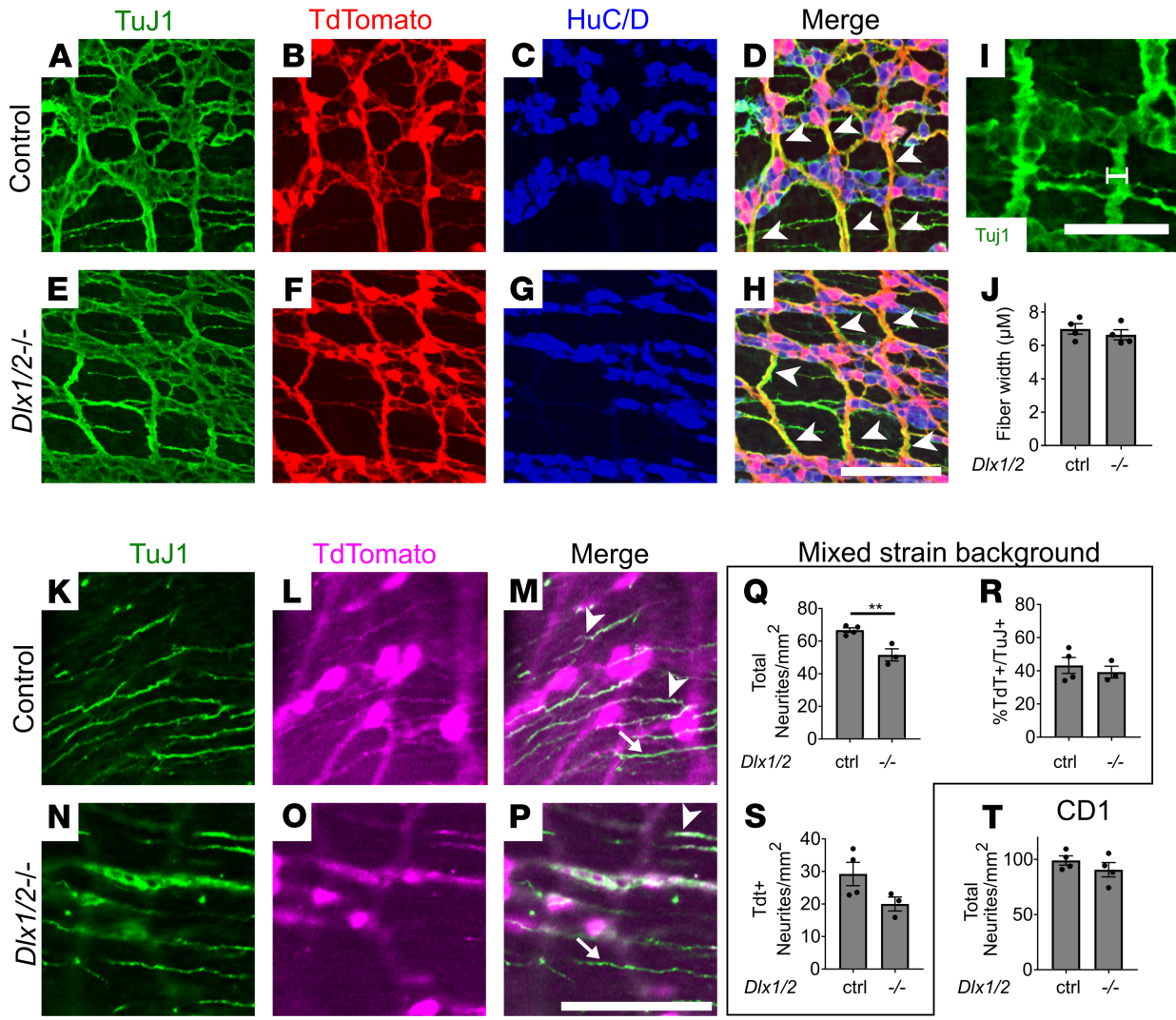

Figure 9. $D I \times 1 / 2^{-/-}$mice have apparently normal large nerve fiber bundles but decreased CSM neurite density on some strain backgrounds. (A-H) Myenteric plexus labeled with Tul1 (green; A and E), TdTomato (red; B and F), and HuC/D (blue; $\mathbf{C}$ and $\mathbf{C}$ ). All large nerve fiber bundles (white arrowheads) were TdTomato+. (I and J) Myenteric plexus nerve fiber bundle width (white line, I) was similar in $D / x 1 / 2^{-1-}$ and control mice (J; $P=0.4565$, Student's $t$ test, $n=4$ per genotype). (K-P) Circular muscle neurites labeled with TuJ1 (green; $\mathbf{K}$ and $\mathbf{N}$ ) and TdTomato (magenta; $\mathbf{L}$ and $\mathbf{O}$ ) from PO bowel. Some neurites were TdTomato and Tuj $1^{+}$(white arrowheads), while others were Tuj $1^{+}$alone (white arrows). (Q) Density of total TuJ $1^{+}$neurites was decreased in DIx1/2 $2^{-1-}$ Vip-IRES-Cre TdTomato PO bowel $(P=0.0077$, Student's $t$ test, $n=4$ [ctrl], $n=$ $3[-/-])$. (R) Ratio of TdTomato ${ }^{+}$neurites to Tuj $1^{+}$neurons was unchanged $(P=0.5587$, Student's $t$ test, $n=4$ [ctrl], $n=3$ $[-/-])$. (S) Density of TdTomato neurites was not statistically different for control and $D / x 1 / 2^{-/-}$mice $(P=0.1006$, Student's $t$ test, $n=4$ [ctrl], $n=3[-/-])$. (T) Density of total Tuj $1^{+}$neurites was normal for DIx1/2-/- mice on a CD1 background $(P=0.3193$, Student's $t$ test, $n=4$ [ctrl], $n=4[-/-])$. Scale bars: $100 \mu \mathrm{m}(\mathbf{D}-\mathbf{H}$ and $\mathbf{K}-\mathbf{P})$ and $50 \mu \mathrm{m}(\mathbf{I}) .{ }^{* *} P<0.001$.

fibers was significantly reduced in $D l \times 1 / 2^{-/-}$mice relative to WT (Figure $10 \mathrm{~K}$ ), demonstrating dramatic reductions in VIP protein in myenteric plexus nerve fibers. Moreover, the relative area of nerve fiber bundles with high VIP expression (likely representing concentrated collections of VIP, i.e., varicosities) was even more dramatically decreased in $D l x 1 / 2^{--}$mice (Figure $10, \mathrm{~L}-\mathrm{N}$ ).

We used similar methods to determine if VIP within nerve cell bodies was reduced. Average VIP intensity within soma was not significantly changed in $D l x 1 / 2^{-1-}$ mice (Figure $10, \mathrm{O}-\mathrm{Q}$ ). However, the relative area of soma with high VIP expression was significantly decreased in Dlx1/2-/ mice (Figure 10, R-T). Together, these results confirm reduced VIP protein in Dlx1/2-- ENS.

\section{Discussion}

Bowel motility disorders include life-threatening problems like Hirschsprung disease where the ENS is missing from distal bowel (33) and neuropathic CIPO where ENS is present, but dysfunctional (1). Human CIPO causes abdominal distension and bowel dysmotility that resembles bowel problems in $D l \times 1 / 2^{--}$mice, but anatomic defects are often difficult to identify in CIPO. Although it is possible that more careful analysis of ENS structure would identify defects in human CIPO bowel, functional defects can also occur in the absence of anatomic abnormalities. For $D l \times 1 / 2^{-/}$mice, we eventually uncovered anatomic defects, but only after RNA-seq identified reduced Vip levels in mutant ENS. RNA-seq is possible in human ENS and might also be valuable in analysis of bowel from people with $\operatorname{CIPO}(34,35)$. 

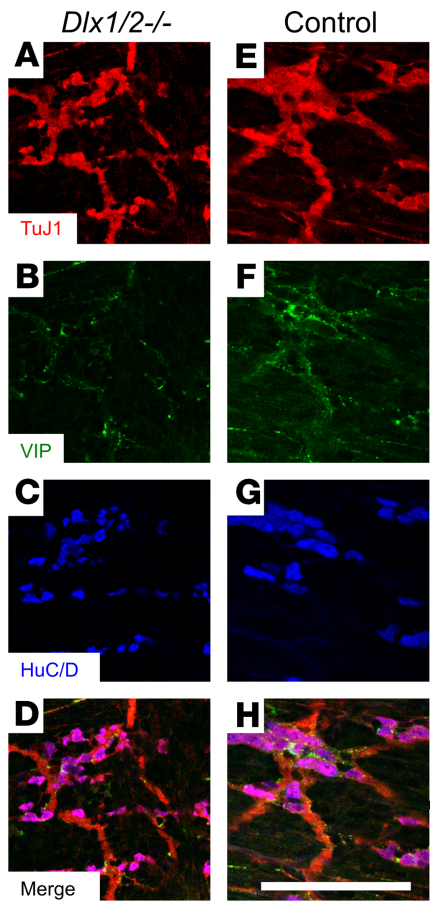

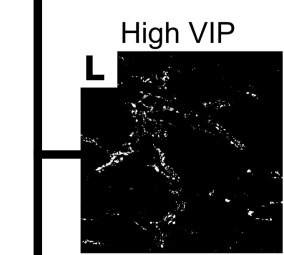

Cell Mask
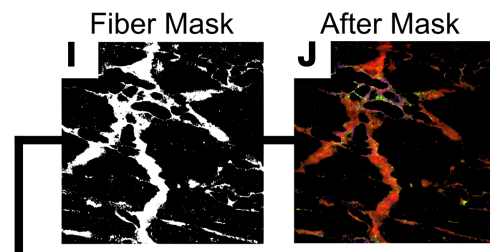

Total area

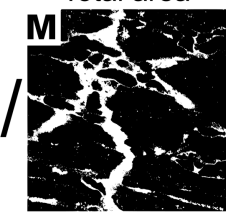

After Mask

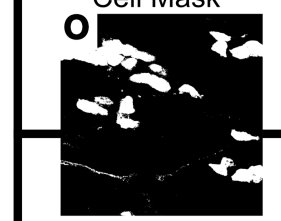

High VIP

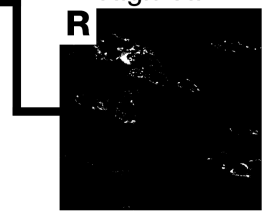

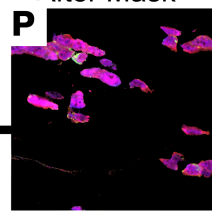

Total area

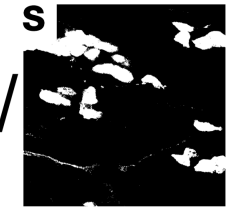

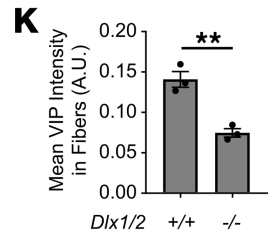

$\mathbf{N}$
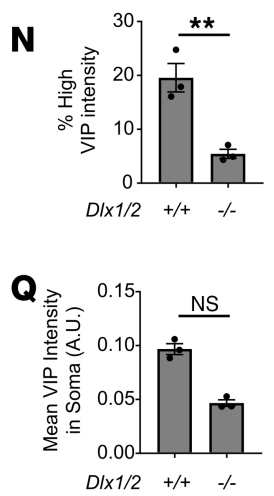

$\mathbf{T}$

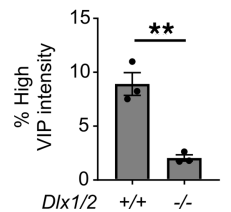

Figure 10. VIP is reduced in DIx1/2-/- nerve fibers and cell bodies. (A-H) High-resolution slices from confocal Z-stacks showing TuJ1 (red), HuC/D (blue), and VIP (green). (I and J) Example mask constructed by thresholding on the TuJ1 channel and subtracting thresholded HuC/D. (K) Average VIP intensity within masked regions was significantly reduced in $D / \times 1 / 2^{-/-}$mutant mice compared with control, suggesting reduced VIP expression in nerve fibers $(P=0.0039$, Student's $t$ test, $n=3[+/+], n=3[-/-])$. (L-N) Total area of pixels with high VIP (defined arbitrarily as VIP intensity $>0.2 \mathrm{AU} ; \mathbf{L}$ ) was divided by total neurite area (M) to determine the percentage of nerve fiber bundles with high VIP expression, an approximation for density of VIP varicosities. This value was significantly reduced in $D / x 1 / 2^{-1-}$ nerve fibers $(P=0.0070$, Student's $t$ test, $n=3[+/+], n=3[-/-] ; \mathbf{N})$. (O and $\mathbf{P})$ Example mask constructed by thresholding on the HuC/D channel. (Q) Average VIP intensity within soma was not significantly decreased in $D / x 1 / 2^{-/-}$mice relative to controls $(P=0.100$, Mann-Whitney rank-sum test, $n=3[+/+], n=3[-/-])$. (R-T) Percentage soma area with high VIP expression was significantly decreased in $D / \times 1 / 2^{-/-}$mice $(P=0.0033$, Student's $t$ test, $n=3(+/+), n=3[-/-]){ }^{* *} P<0.001$. NS, not significant.

We chose to investigate bowel motility in $D l x 1$ and $D l x 1 / 2$ mutants because $D l x 1$ and $D l x 2$ are expressed in developing ENS at ages relevant for ENCDC migration, differentiation, and subtype specification, and because the original description of $D l \times 2^{-/-}$mice suggested they die from bowel dysmotility $(10,17-20)$. We showed that neonatal $D l \times 1 / 2^{-/-}$mice have profound bowel function defects, including delayed gastric emptying and slow SI transit via an in vivo FITC-dextran assay. FITC-dextran was found in the stomachs of all Dlx $1 / 2^{-/-}$mice tested, confirming that despite cleft palate, $D l \times 1 / 2^{-/-}$mutants were capable of swallowing and that their stomachs empty slowly. When we maintained bowel in an oxygenated organ bath and treated with TTX, which blocks nerve cell activity, we found that $D l \times 1 / 2^{-/-}$bowels had severely reduced TTX-sensitive contraction patterns. Interpreting these findings is challenging because a complex interplay of excitatory and inhibitory neural input regulates bowel smooth muscle contraction and relaxation. A naive interpretation is that neuron-mediated smooth muscle excitation (contraction) is defective in $D l \times 1 / 2^{-/-}$mice. Another possibility is that $D l \times 1 / 2^{-/-}$bowel has reduced smooth muscle inhibition at baseline, leading to a mildly contracted phenotype approaching that of control bowel treated with TTX. Consistent with this hypothesis, $D l x 1 / 2^{-1-}$ bowel did not seem to contract significantly upon TTX treatment, unlike control bowel. Moreover, bowel videos suggest that neurally mediated "contraction complexes" actually consist of complex motor patterns, where bowel first straightens (possibly reflecting longitudinal smooth muscle relaxation), then contracts rapidly, and finally returns to baseline tortuosity (Supplemental Video 1). TTX-treated bowel, which is highly contracted, never undergoes this pattern, and $D l \times 1 / 2^{-/-}$mouse bowel does so only rarely. Because $D l \times 1 / 2^{-1-}$ mice had a normal pattern of TTX-insensitive (i.e., non-neuronal) contractions, and Dlx1 and Dlx2 are 
prominently expressed in developing ENS, we interpret these findings to mean that $D l \times 1 / 2$ mutations cause neuropathic CIPO-like disease in mice.

Surprisingly, immunohistochemical studies showed $D l \times 1 / 2^{-/-}$bowel had largely normal ENS structure at birth, with normal neuronal and glial density and normal ratios of many enteric neuronal subtypes, even though changes in these parameters have been identified in many mouse lines $(3,36-42)$. We note, however, that our extensive anatomic studies were not exhaustive, that some antibodies gave weak signals in P0 ENS (e.g., tyrosine hydroxylase, substance P), and that we did not perform enough replicates to define sex-restricted defects. Furthermore, although $D l \times 2^{-/-}$mice had a small ENCDC migration delay at E12.5, this may occur because of different strain backgrounds for $D l \times 2^{-/}(\mathrm{C} 57 \mathrm{BL} / 6)$ and $D l \times 1 / 2^{-/-}$mice (CD1). Strain effects on ENCDC migration have been reported in the ENS (29). It is, however, unlikely that this minimal transient ENCDC migration delay impairs bowel function at P0.

To identify defects causing CIPO-like disease in $D l x$-mutant mice, we performed RNA-seq. Unexpectedly, we identified a potentially novel connection between $D l x$ genes and Vip. The $D l x 1 / 2^{-1-}$ ENS had decreased Vip mRNA at P0 (53\% reduction) and a greater reduction in Vip mRNA at E14.5 (64\% reduction by qRT-PCR, $84 \%$ reduction by RNA-seq). Consistent with the RNA data, the intensity of VIP immunostaining in cell bodies and in neurites was significantly reduced in $D l \times 1 / 2^{-1-}$ myenteric plexus. Moreover, Dlx $1 / 2^{-1-}$ VIP-IRES-Cre TdTomato reporter mice had significantly fewer TdTomato ${ }^{+}$neurons in myenteric $(18.4 \%)$ and submucosal plexus (51.5\%), even though we could not appreciate neuron loss via VIP immunohistochemistry. We attribute this difference to limited VIP protein in nerve cell bodies, which may make neuron counting less reliable with antibody staining compared to using Vip-lineage reporter mice.

In the ENS, VIP acts as a critical ENS neurotransmitter with multiple roles. In myenteric plexus, VIP is coexpressed with NOS and PACAP in inhibitory motor neurons. These neurons inhibit the multicellular motor syncytium composed of smooth muscle cells, interstitial cells of Cajal, and platelet-derived growth factor receptor $\alpha^{+}\left(\mathrm{PDGFR} \alpha^{+}\right)$cells (5). In submucosal plexus, $\mathrm{VIP}^{+}$secretomotor and vasodilator neurons influence epithelial secretion and blood flow, respectively. VIP also acts on neurons and glia within the ENS (43) and is an important modulator of gut immunity (44). Thus, there are many ways that altered VIP abundance might impact gut motility, epithelial function, and immune system activity to impair $D l \times 1 / 2^{-/-}$mouse survival. The importance of VIP in ENS function is highlighted by the observation that Vip ${ }^{-/}$mice have severely delayed SI transit, impaired mucin production, smooth muscle thickening, and shortened bowel, and die early due to GI stenosis (45). In contrast, $\mathrm{Vip}^{+/-}$mice are healthy enough to reproduce, but limited data exist about bowel motility in Vip heterozygotes. These observations are consistent with the hypothesis that reduced VIP levels in $D l \times 1 / 2^{-/-}$mice could contribute to bowel dysmotility, but it is not easy to determine if the level of VIP reduction detected in $D l \times 1 / 2^{-/-}$mice adequately explains the profound bowel motility defects in these animals. We found altered levels of many additional mRNAs in the ENS of Dlx $1 / 2^{-1-}$ mice and expression changes in 1 or more of these genes might also contribute to bowel dysmotility.

Our findings highlight striking differences between the role of $D l x 1$ and $D l x 2$ in the ENS and their developmental functions in the brain. In the CNS, Dlx 1 and $D l x 2$ are required for migration of multiple populations of inhibitory interneuron precursors from the ventral telencephalon into the neocortex and olfactory bulb $(13,14,46)$. Dlx2 also regulates expression of the transcription factor $Z f h x 1 b$ in the brain (21). Zfhx $1 b$ is critical for normal ENCDC migration, and in humans $Z F H X 1 B$ mutations cause Mowat-Wilson syndrome (a genetic disorder characterized by intellectual disability, epilepsy, microcephaly, and Hirschsprung disease) $(22,30,47)$. Given this substantial evidence implicating $D l x 1$ and $D l x 2$ in processes critical for neuronal migration and differentiation, we were surprised that $D l \times 1 / 2^{-/-}$bowel exhibited normal ENCDC migration at E12.5 and normal neuronal density at $\mathrm{P} 0$.

In CNS, Dlx 1 and Dlx2 are necessary for expression of glutamic acid decarboxylase 1 and 2 (Gad1 and Gad2), which catalyze GABA synthesis from glutamate $(48,49)$. Surprisingly, we found normal numbers of GABAergic neurons in Dlx1/2-/- ENS, and we did not observe significantly decreased Gad1 or Gad2 gene expression in $D l x$-mutant ENCDCs at E14.5 or P0. These findings suggest that in the ENS, GABAergic neurons do not depend on $D l x 1$ or Dlx2 for Gad1, Gad2, or GABA synthesis. (Interestingly, our GABA antibody labeled more neurons than were previously reported in the literature $[53 \%$ vs. $5 \%$ to $8 \%$ of myenteric neurons; refs. 40, 41]. We suspect this is because we quantified both brightly [ 11\%] and dimly labeled $[\sim 42 \%]$ populations of $\mathrm{GABA}^{+}$neurons [Figure $\left.6 \mathrm{X}\right]$, whereas prior studies may have only quantified brightly stained neurons.) We also note that loss of $D l x 1$ causes apoptosis in subsets of CNS interneurons in 1- to 2-month-old mice (15), but Dlx1 adult ENS showed no evidence of neuronal subtype 
loss or decreased neuronal density. Together, these findings underscore highly distinct roles for $D l x$ genes in enteric neurons, compared with the CNS.

Summary. CIPO describes a poorly understood constellation of diseases in which bowel motility is abnormal despite the presence of neurons throughout the bowel. Although neuronal dysfunction accounts for many cases of CIPO, few causative genes have been identified $(1,5,50)$. Our study provides an example of how bowel motility defects may occur in the absence of easily identified ENS structural defects, likely due to gene-level dysregulation. To our knowledge, few prior studies have performed such a comprehensive analysis of the neonatal ENS in mutant mice. There is a strong need to develop new tools to assess motility at young ages, since many ENS-relevant genes are also critical for palate development (12, 20, 51), kidney development (52-54), and other systems essential for life after birth. Our study is also the first to our knowledge to comprehensively investigate the role of DLX1 and DLX2 in gut motility, and we believe the first to show a regulatory link between DLX1 and DLX2 and Vip. Remarkably, well-described functions for Dlx1 and Dlx2 in CNS, like control of cell migration and regulation of Gad1 and Gad2 to produce GABA, do not appear to occur in the ENS. Furthermore, DLX2 was reported to be essential for CNS Zfhx1b expression, and mutations in Zfhx $1 b$ lead to almost complete loss of enteric neurons in mice, whereas bowel colonization by ENCDCs proceeds normally in $D l \times 1 / 2^{-1-}$ mice. Collectively these observations highlight how differently genes shared between ENS and CNS may function. Future studies in humans are important to determine if Dlx mutations are present in individuals with CIPO and to elucidate if $D l x$ genes control Vip expression in developing human ENS.

\section{Methods}

Animals. All mouse experiments were performed in accordance with the Children's Hospital of Philadel-

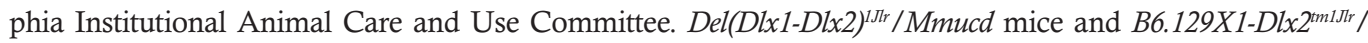
Mmucd mice (referred to as $D l \times 1 / 2$ and $D l \times 2$ mice, respectively) $(10,12)$ were obtained from the MMRRC (RRID:MMRRC_036673-UCD and RRID:MMRRC_015870-UCD) and were maintained on a

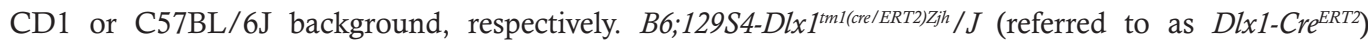
mice were ordered from the Jackson Laboratory (RRID:IMSR_JAX:014551) and were maintained on a C57BL/6J background. EDNRB-EGFP-L10a mice (32) were bred with Dlx1/2 mice and maintained on a mixed C57BL6/J $\times$ CD1 background. ChAT-EGFP-L10a mice (RRID:IMSR_JAX:030250; C57BL/6J) were a gift from Joseph Dougherty at Washington University School of Medicine, St. Louis, Missouri, USA. Dlx1/2 ChAT-EGFP-L10a mice were obtained by breeding homozygous ChAT-EGFP-L10a mice to Dlx1/2 heterozygotes and were maintained on a mixed C57BL/6J $\times$ CD1 background. Dlx1/2 Ret mice were generated by breeding $D l \times 1 / 2$ mice to $\operatorname{Ret}^{T G M}$ mice (referred to as Ret mice; C57BL/6J) (55) and were maintained on a C57BL/6J $\times$ CD1 background. Tg(Wnt1cre)11Rth mice (referred to as Wnt1-Cre; RRID:IMSR_JAX:003829) and Gt(ROSA)26Sortm9(CAGtdTomato)Hze mice (referred to as $R 26 R-T d$ Tomato; RRID:IMSR_JAX:007909) were obtained from the Jackson Laboratory. Wnt1-Cre R26R-TdTomato mice on a mixed (C57BL/6J $\times \mathrm{CBA} / \mathrm{J})$ background were bred to $D l \times 1 / 2$ mice and maintained on a mixed $(\mathrm{C} 57 \mathrm{BL} / 6 \mathrm{~J} \times \mathrm{CD} 1 \times \mathrm{CBA} / \mathrm{J}) \mathrm{F} 1$ background. Vip ${ }^{\text {tml(cre)Zjh }} / J$ mice (referred to as Vip-IRES-Cre; RRID:IMSR JAX:010908) on a mixed C57BL/6 × 129S4 background were bred to Dlx1/2 and R26R-TdTomato mice and maintained on a mixed C57BL/6J $\times$ CD1 $\times 129 \mathrm{~S} 4$ background. Genotyping was performed using previously published and novel primers (Supplemental Table 1). Vaginal plug day was considered E0.5.

Preparation of whole gut samples. Adult mice were euthanized in $\mathrm{CO}_{2}$ (5 minutes) and cervically dislocated. P0 mice were euthanized by decapitation. Bowel was placed in cold PBS, opened along the mesenteric border, and pinned onto Sylgard 184 Silicone Elastomer (Dow Corning), serosa up, using insect pins. Tissue was fixed in $4 \%$ paraformaldehyde $\left(20-30\right.$ minutes, $\left.25^{\circ} \mathrm{C}\right)$ and transferred to PBS. Muscle was peeled from the mucosa and submucosa to separate myenteric and submucosal plexus. Peeled gut was equilibrated at room temperature for 30 minutes in $50 \%$ glycerol $/ 50 \%$ PBS and stored $\left(-20^{\circ} \mathrm{C}\right)$ until staining.

To prepare E12.5 bowel, pregnant dams were euthanized in $\mathrm{CO}_{2}$ (5 minutes) and embryos dissected in Leibovitz's L-15 medium (Thermo Fisher Scientific, 41300039). Bowel was fixed in 4\% paraformaldehyde (30 minutes), equilibrated at room temperature (30 minutes) in $50 \%$ glycerol $/ 50 \% \mathrm{PBS}$, and stored $\left(-20^{\circ} \mathrm{C}\right)$ until staining.

E12.5 neuron culture. Pregnant dams were euthanized $\left(\mathrm{CO}_{2}, 5\right.$ minutes) and E12.5 embryos were dissected in Leibovitz's L-15 medium. Whole bowel was incubated in Dispase $(0.2 \mathrm{mg} / \mathrm{mL}$; Thermo Fisher Scientific, $17105-041)$ and collagenase $\left(0.2 \mathrm{mg} / \mathrm{mL}\right.$; Sigma-Aldrich, C-6885) in PBS (5 minutes, $\left.37^{\circ} \mathrm{C}\right)$, rinsed with cold PBS using a cell strainer, dissociated by triturating 35 times through a P1000 pipet, and then centrifuged and resuspended in Neurobasal Media (Invitrogen, C21103049) supplemented with L-glutamine 
(2 mM; Invitrogen, 25030081), B27 (1×; Invitrogen, 12587010), penicillin/streptomycin (1×; Invitrogen, 15140122 ), and $50 \mathrm{ng} / \mathrm{mL} 6 \times$ His-GDNF prepared as described previously (56). Cells were plated on Nunc Lab-Tek 8-well chamber slides (Thermo Fisher Scientific, 177402) coated overnight with $100 \mu \mathrm{g} / \mathrm{mL}$ poly-Dlysine (Sigma-Aldrich, P7280) and $20 \mu \mathrm{g} / \mathrm{mL}$ laminin (VWR, 47743-682) for 2 hours. After 2 days, cultures were fixed (15 minutes, $4 \%$ paraformaldehyde) and immunostained.

Immunofluorescent and enzymatic staining of whole-mount bowel. Whole-mount bowel stored in 50\% glycerol $/ 50 \%$ PBS at $-20^{\circ} \mathrm{C}$ was rinsed in PBS. Fixed E12.5 bowel was maintained in 48-well plates for the duration of immunostaining. Samples were blocked (2 hours) in PBS plus $0.1 \%$ or $0.5 \%$ Triton X-100 (PBST) with 5\% normal donkey serum (NDS) (Jackson Immuno Research Laboratory) and incubated in primary antibody with gentle rocking at $4^{\circ} \mathrm{C}$ overnight, except for HuC/D (ANNA-1) antibody, where we incubated tissues for 2 hours at room temperature. Primary antibodies are in Supplemental Table 2. Tissues were washed 3 times, 5 minutes each, in PBST and incubated in secondary antibody (Supplemental Table 2 ) at room temperature with gentle rocking (1 hour). After 3 additional 5-minute washes in PBS, samples were mounted serosal side up in $50 \%$ glycerol $/ 50 \%$ PBS on glass slides. NADPH diaphorase staining was performed as described previously (57). Briefly, NADPH (Sigma-Aldrich) and Nitro Blue Tetrazolium were dissolved in PBS with $0.2 \%$ Triton X-100. Samples were incubated in this solution $\left(7-15\right.$ minutes, $37^{\circ} \mathrm{C}$ ), rinsed in PBS, and then stained with antibodies as described above. For tissue labeled with anti-ChAT antibody, samples were fixed in 100\% methanol on ice (30 minutes) and placed in Dent's bleach (1:4:1 100\% DMSO/100\% methanol/30\% $\mathrm{H}_{2} \mathrm{O}_{2}$ ) for 1 hour at room temperature before blocking.

Immunofluorescent staining of cultured cells. Slides were rinsed in PBS, blocked ( 1 hour in PBS $+0.1 \%$ or $0.5 \%$ Triton X-100 [PBST) with 5\% NDS), and incubated with primary antibody (Supplemental Table 2) overnight $\left(4^{\circ} \mathrm{C}\right)$. Tissues were washed 3 times, 5 minutes each, in PBS and incubated in secondary antibody (Supplemental Table 2) for 1 hour (room temperature). After 3 additional 5-minute washes, samples were mounted in $50 \%$ glycerol $/ 50 \%$ PBS plus DAPI or Vectashield.

Microscopy. Zeiss Axio Imager.A2, Axio Observer.A1, or LSM 710 microscopes and Zeiss Zen software were used to acquire images. Confocal images show single optical projections or maximum intensity projections as indicated in figure legends. For image processing, ImageJ (NIH) and Adobe Photoshop were used to rotate, crop, and uniformly color adjust images.

Quantitative analysis of antibody-stained bowel. Unless otherwise specified, 5 to 10 randomly selected 20x fields (a $0.045 \mathrm{~mm}^{2}$ region) per sample were analyzed for all quantifications. Using whole-mount stained samples, we counted the number of enteric neurons $\left(\mathrm{HuC} / \mathrm{D}^{+}\right.$cells), enteric glia (SOX $10^{+} \mathrm{S} 100 \beta^{+}$cells), and SOX $10^{+}$cells (a mix of glia and ENS precursors) to determine density. Enteric neuron subtype proportions were quantified by counting the number of $\mathrm{HuC} \mathrm{D}^{+}, \mathrm{GFP}^{+}$, neuronal $\mathrm{NOS}^{+}\left(\mathrm{nNOS}^{+}\right)$, calretinin ${ }^{+}$, NADPH-diaphorase ${ }^{+}, \mathrm{GABA}^{+}, \mathrm{VIP}^{+}$, or somatostatin ${ }^{+}$cells. Neurite fiber density was quantified drawing a line perpendicular to CSM and counting the number of neurites that crossed the line. Observers were blinded to genotype for all quantitative analyses.

For measuring VIP intensity, we used 5 to 10 randomly selected $63 \times$ confocal $Z$-stacks per sample. Only slices capable of being accurately thresholded were analyzed. Using CellProfiler (58), images were thresholded with the Global with Minimum Cross Entropy settings and masked to quantify VIP intensity within $\mathrm{HuC} / \mathrm{D}^{+}$cell bodies and in $\mathrm{HuC} / \mathrm{D}-\mathrm{TuJ} 1^{+}$nerve fibers.

Quantitative analysis of neurites in culture. One large $\left(>12 \mathrm{~mm}^{2}\right)$ image was taken within a well (2 separate wells per embryo). Neurites from intact cells were traced, and cell bodies were counted using the Simple Neurite Tracer plugin in ImageJ by investigators blinded to genotype. Because neurites sometimes crossed, making tracing difficult, we determined average total neurite length per cell by dividing the sum of all neurite lengths by the number of nerve cell bodies. When neurites could be traced unambiguously, we measured the length of all neurites, added these lengths together to determine total neurite length, and plotted the data in a histogram.

Delivery by Cesarean section. Pregnant mouse mothers (E18.5) were injected subcutaneously with $2 \mathrm{mg}$ of progesterone (Sigma-Aldrich, P3972-5G). The next morning (E19.5), mice were euthanized via cervical dislocation. Pups were removed from the mother as previously described (59) and placed on a warming pad (DCT-20, Kent Scientific).

Intestinal transit assay. A P0 FITC-dextran intestinal transit assay was performed as previously described (60), with 2 modifications: (a) a total of 3 hours passed between time of feeding and time of dissection, and (b) bowel was cut into 10 segments (esophagus, stomach, 6 SI pieces, cecum, and colon). 
P35 mice were deprived of food for 14 hours and gavage-fed $100 \mu \mathrm{L}$ of $10 \mathrm{mg} / \mathrm{mL}$ FITC-dextran (70 $\mathrm{kDa}$ ) plus $2 \%$ methylcellulose. After 1 hour, mice were euthanized $\left(\mathrm{CO}_{2}\right)$ and whole bowel was dissected, cut into 16 segments (stomach, 10 SI, 2 cecum, and 3 colon pieces), and placed in Eppendorf tubes containing $400 \mu \mathrm{L}$ PBS. Tubes were vortexed and centrifuged ( $4000 \mathrm{~g}, 10$ minutes). Fifty microliters of supernatant was combined with $50 \mu \mathrm{L}$ of PBS and analyzed using a fluorometer.

$R N A$ extraction. E14.5 dams were euthanized with $\mathrm{CO}_{2} . \mathrm{P} 0$ pups were delivered by Cesarean section as described above. Pups were rapidly removed from the mother. E14.5 SI was dissected in ice-cold Leibovitz's L-15 medium (Life Technologies, 41300039$)$ and dissociated $\left(15\right.$ minutes, $\left.37^{\circ} \mathrm{C}\right)$ in Dispase $(0.2 \mathrm{mg} / \mathrm{mL}$; Thermo Fisher Scientific, 17105-041) and collagenase (0.2 mg/mL; Sigma-Aldrich, C-6885) in PBS with P1000 trituration. P0 SI was dissected in Leibovitz's L15 medium, snipped into small pieces using insulin needles (Becton Dickinson, 08290-3284-18), and dissociated (30 minutes, $37^{\circ} \mathrm{C}$ ) in Liberase (Sigma-Aldrich, 5401135001) supplemented with DNase I (Roche, 04716728001), $6 \mathrm{mM} \mathrm{MgCl}$ and $1 \mathrm{mM} \mathrm{CaCl}{ }_{2}$ in $\mathrm{HBSS}$ with repeated P1000 trituration. Fluorescent EGFP-L10a ${ }^{+}$cells (E14.5) or Tdtomato ${ }^{+}$cells (P0) were sorted on a BD FACSJazz and collected in Neurobasal medium (Invitrogen, 21103049). RNA was extracted using the RNeasy Plus Micro kit (Qiagen, 74034) combined with the RNase-Free DNAse Set (Qiagen, 79254). Samples were run on an Agilent Bioanalyzer and used if RNA integrity number (RIN) was greater than 7.0.

Bowel physiology. Between 1 and 3 hours after Cesarean section, pups were decapitated. Bowel was removed and placed in warmed $\left(37^{\circ} \mathrm{C}\right)$, oxygenated $\left(95 \% \mathrm{O}_{2}, 5 \% \mathrm{CO}_{2}\right)$ Krebs-Ringers solution $(118 \mathrm{mM} \mathrm{NaCl}, 4.6 \mathrm{mM}$ $\mathrm{KCl}, 2.5 \mathrm{mM} \mathrm{CaCl}_{2}, 1.2 \mathrm{mM} \mathrm{MgSO}_{4}, 1 \mathrm{mM} \mathrm{NaH}_{2} \mathrm{PO}_{4}, 25 \mathrm{mM} \mathrm{NaHCO}_{3}, 11 \mathrm{mM}$ D-glucose, pH 7.4) in a horizontal organ bath (Hugo Sachs Elektronik, Harvard Apparatus, D-79232) with fluid continually replaced at a rate of $6.15 \mathrm{~mL} / \mathrm{min}$. Proximal SI was isolated by making 1 cut distal to the stomach and a second cut approximately $2 \mathrm{~cm}$ proximal to the cecum. The proximal end of the SI was cannulated and tied to a P2/P10 Ultra Micro Pipet Tip (Thermo Fisher Scientific, 02-707-438) connected using an L-shaped connector (Harvard Apparatus, 72-1407) and tubing to an upright $3 \mathrm{~mL}$ syringe (Becton Dickinson, 309657). Fluid level in the syringe was maintained at $1.3 \mathrm{~cm}$ above the proximal end of the bowel. Proximal and distal bowel were at the same elevation. Bowel was pinned loosely on a secure piece of Sylgard 184 Silicone Elastomer (Dow Corning), illuminated to contrast with background, and filmed with a camera (Olympus, PEN Mini E-PMI) connected to a dissecting microscope (Olympus SZ-PT SZ40). After an initial 20-minute video, $1 \mathrm{mM}$ TTX stock solution (Abcam, ab120054) dissolved in sodium citrate buffer ( $40 \mathrm{mM}$ citric acid monohydrate and $60 \mathrm{mM}$ trisodium citrate dihydrate, $\mathrm{pH} 4.8$ ) was diluted in Krebs-Ringer to a concentration of $1 \mu \mathrm{M}$. The resulting solution was oxygenated and pumped into the organ bath, and then another 20-minute video was recorded.

Analysis of bowel motility patterns. Videos were converted to .wmv format using Movie Maker and saved at $1 \times$ and $16 \times$ speeds. In-house MATLAB (MathWorks) scripts (http://github.com/christinawright100/ BowelSegmentation) were used to threshold the movies, generate kymographs, and perform Fourier transform analysis. Peak frequency was determined by calculating the Fourier transform at each vertical slice of the kymograph, averaging these, and plotting the averages to determine the peak frequency. Investigators blinded to genotype and condition quantified neurally mediated contractions in kymographs and recorded if contractions were rhythmic or nonrhythmic. To quantify bowel width and tortuosity, 10 random frames were saved from each video and analyzed in ImageJ. For each frame, we measured 5 random widths (randomly generated using a MATLAB script) along the proximal bowel (50 widths per bowel). For tortuosity, total bowel length was divided by straight-line distance.

$q R T-P C R$ analysis. qRT-PCR was performed using previously described primers that we validated (Supplemental Table 3 and Supplemental Figure 4) and SSoFast Evagreen (Bio-Rad, 172-5211). Fold change relative to control was computed using the $\Delta \Delta \mathrm{Ct}$ method with normalization to Gapdh mRNA levels.

$R N A$-seq. RNA was extracted as described above. cDNA libraries were generated from samples with RIN greater than 7.0 using the SMARTer Stranded Total RNA-Seq Kit v2 Pico Input Mammalian (Takara Bio, 634411) designed to work with very-low-input RNA. Libraries were sequenced on an Illumina HiSeq 2500 to a read depth of 17 to 26 million paired-end reads per sample. Reads were mapped to the genome using the STAR RNA-seq aligner (61), normalized using Pipeline of RNA-seq Transformations (PORT) (62), and analyzed in R (R Foundation) using Limma's Voom method (63, 64). Data sets will be made available in the NCBI's GEO database pending publication of this paper (GEO accession GSE145362).

Statistics. We used Prism 7.03 (GraphPad Software) and SigmaPlot 11.0 (Systat Software) for statistical analysis. A 2-sided Student's $t$ test or Mann-Whitney rank-sum test was used when comparing 2 groups. When comparing multiple groups, a 1-way ANOVA with Tukey's post hoc multiple-comparisons tests 
was used unless assumptions were not met, in which case we used a Kruskal-Wallis test with Dunn's post hoc multiple-comparisons test. When comparing weight across multiple time points and groups, a 2-way repeated-measures ANOVA was used with Tukey's post hoc multiple-comparisons tests. A cutoff of $P<$ 0.05 was considered significant. For RNA-seq analyses, a false discovery rate-adjusted $P$ value (i.e., $q$ value) of 0.10 was considered significant. Data represent mean \pm SEM. Investigators were blinded to condition for all quantifications.

Study approval. All animal experiments were approved by the Children's Hospital of Philadelphia IACUC.

\section{Author contributions}

CMW and ROH designed experiments, analyzed results, and wrote the manuscript. CMW, JPG, HLM, DRK, SS, and BAM performed experiments. All authors edited the manuscript.

\section{Acknowledgments}

We are grateful to Marina Avetisyan, Silvia Huerta López, Joel Bornstein, and John Grider for insightful comments and assistance during preparation of the manuscript. This work was supported by the Irma and Norman Braman Endowment (to ROH), the Suzi and Scott Lustgarten Center Endowment (to ROH), the Children's Hospital of Philadelphia Research Institute (to ROH), Children's Discovery Institute of Washington University and Saint Louis Children's Hospital (MD-II2013-269) (to ROH), NIH grants 5 F30 DK117546-02 (to CMW) and R01 DK087715 (to ROH), March of Dimes 6-FY15-235 (to ROH), and the Burroughs Wellcome Fund Clinical Scientist Award in Translational Research (1008525) (to ROH).

Address correspondence to author: Robert O. Heuckeroth, Children's Hospital of Philadelphia Research Institute, 3615 Civic Center Boulevard, Abramson Research Center, Suite 1116i, Philadelphia, Pennsylvania 19104-4318, USA. Phone: 215.590.1209; Email: HeuckerothR@email.chop.edu.

1. Antonucci A, et al. Chronic intestinal pseudo-obstruction. World J Gastroenterol. 2008;14(19):2953-2961.

2. Ueno T, Fukuzawa M. Current status of intestinal transplantation. Surg Today. 2010;40(12):1112-1122.

3. Lake J, Heuckeroth RO. Enteric nervous system development: migration, differentiation, and disease. Am J Physiol Gastrointest Liver Physiol. 2013;305(1):G1-24.

4. Espinosa-Medina I, et al. Dual origin of enteric neurons in vagal Schwann cell precursors and the sympathetic neural crest. Proc Natl Acad Sci USA. 2017;114(45):11980-11985.

5. Schneider S, Wright CM, Heuckeroth RO. Unexpected roles for the second brain: enteric nervous system as master regulator of bowel function. Annu Rev Physiol. 2019;81:235-259.

6. De Giorgio R, et al. Chronic intestinal pseudo-obstruction related to viral infections. Transplant Proc. 2010;42(1):9-14.

7. Pingault V, et al. SOX10 mutations in chronic intestinal pseudo-obstruction suggest a complex physiopathological mechanism. Hum Genet. 2002;111(2):198-206.

8. Van Goethem G, Schwartz M, Löfgren A, Dermaut B, Van Broeckhoven C, Vissing J. Novel POLG mutations in progressive external ophthalmoplegia mimicking mitochondrial neurogastrointestinal encephalomyopathy. Eur J Hum Genet. 2003;11(7):547-549.

9. De Giorgio R, Bianco F, Latorre R, Caio G, Clavenzani P, Bonora E. Enteric neuropathies: Yesterday, today and tomorrow. Adv Exp Med Biol. 2016;891:123-133.

10. Qiu M, et al. Null mutation of Dlx-2 results in abnormal morphogenesis of proximal first and second branchial arch derivatives and abnormal differentiation in the forebrain. Genes Dev. 1995;9(20):2523-2538.

11. Thomas BL, et al. Role of Dlx-1 and Dlx-2 genes in patterning of the murine dentition. Development. 1997;124(23):4811-4818

12. Qiu M, et al. Role of the Dlx homeobox genes in proximodistal patterning of the branchial arches: mutations of Dlx-1, Dlx-2, and D1x-1 and -2 alter morphogenesis of proximal skeletal and soft tissue structures derived from the first and second arches. Dev Biol. 1997;185(2):165-184

13. Anderson SA, Eisenstat DD, Shi L, Rubenstein JL. Interneuron migration from basal forebrain to neocortex: dependence on Dlx genes. Science. 1997;278(5337):474-476.

14. Anderson SA, et al. Mutations of the homeobox genes Dlx-1 and Dlx-2 disrupt the striatal subventricular zone and differentiation of late born striatal neurons. Neuron. 1997;19(1):27-37.

15. Cobos I, et al. Mice lacking Dlx1 show subtype-specific loss of interneurons, reduced inhibition and epilepsy. Nat Neurosci. $2005 ; 8(8): 1059-1068$.

16. Soret R, et al. A collagen VI-dependent pathogenic mechanism for Hirschsprung's disease. J Clin Invest. 2015;125(12):4483-4496.

17. Kapoor A, Auer DR, Lee D, Chatterjee S, Chakravarti A. Testing the Ret and Sema3d genetic interaction in mouse enteric nervous system development. Hum Mol Genet. 2017;26(10):1811-1820.

18. Vohra BP, et al. Differential gene expression and functional analysis implicate novel mechanisms in enteric nervous system precursor migration and neuritogenesis. Dev Biol. 2006;298(1):259-271

19. Visel A, Thaller C, Eichele G. GenePaint.org: an atlas of gene expression patterns in the mouse embryo. Nucleic Acids Res. 2004;32(Database issue):D552-D556. 
20. López SH, et al. Loss of Tbx3 in murine neural crest reduces enteric glia and causes cleft palate, but does not influence heart development or bowel transit. Dev Biol. 2018;444 Suppl 1:S337-S351.

21. McKinsey GL, et al. Dlx1\&2-dependent expression of Zfhx1b (Sip1, Zeb2) regulates the fate switch between cortical and striatal interneurons. Neuron. 2013;77(1):83-98.

22. Van de Putte T, et al. Mice lacking ZFHX1B, the gene that codes for Smad-interacting protein-1, reveal a role for multiple neural crest cell defects in the etiology of Hirschsprung disease-mental retardation syndrome. Am J Hum Genet. 2003;72(2):465-470.

23. Baxter AL, et al. A novel partial duplication of ZEB2 and review of ZEB2 involvement in Mowat-Wilson syndrome. Mol Syndromol. 2017;8(4):211-218.

24. Schill EM, Wright CM, Jamil A, LaCombe JM, Roper RJ, Heuckeroth RO. Down syndrome mouse models have an abnormal enteric nervous system. JCI Insight. 2019;5:124510.

25. Jeong J, Cesario J, Zhao Y, Burns L, Westphal H, Rubenstein JL. Cleft palate defect of Dlx1/2\% mutant mice is caused by lack of vertical outgrowth in the posterior palate. Dev Dyn. 2012;241(11):1757-1769.

26. Roberts RR, et al. The first intestinal motility patterns in fetal mice are not mediated by neurons or interstitial cells of Cajal. J Physiol (Lond). 2010;588(pt 7):1153-1169.

27. Fu M, et al. Vitamin A facilitates enteric nervous system precursor migration by reducing Pten accumulation. Development 2010;137(4):631-640.

28. Lake JI, Tusheva OA, Graham BL, Heuckeroth RO. Hirschsprung-like disease is exacerbated by reduced de novo GMP synthesis. J Clin Invest. 2013;123(11):4875-4887.

29. Walters LC, Cantrell VA, Weller KP, Mosher JT, Southard-Smith EM. Genetic background impacts developmental potential of enteric neural crest-derived progenitors in the Sox10Dom model of Hirschsprung disease. Hum Mol Genet. 2010;19(22):4353-4372.

30. Stanchina L, Van de Putte T, Goossens M, Huylebroeck D, Bondurand N. Genetic interaction between Sox10 and Zfhx1b during enteric nervous system development. Dev Biol. 2010;341(2):416-428.

31. Lomax AE, Furness JB. Neurochemical classification of enteric neurons in the guinea-pig distal colon. Cell Tissue Res. 2000;302(1):59-72.

32. Avetisyan M, et al. Muscularis macrophage development in the absence of an enteric nervous system. Proc Natl Acad Sci USA. 2018;115(18):4696-4701.

33. Heuckeroth RO. Hirschsprung disease - integrating basic science and clinical medicine to improve outcomes. Nat Rev Gastroenterol Hepatol. 2018;15(3):152-167.

34. Bondurand N, Southard-Smith EM. Mouse models of Hirschsprung disease and other developmental disorders of the enteric nervous system: Old and new players. Dev Biol. 2016;417(2):139-157.

35. May-Zhang AA, Deal KK, Southard-Smith EM. Optimization of laser-capture microdissection for the isolation of enteric ganglia from fresh-frozen human tissue. J Vis Exp. 2018;(136).

36. Musser MA, Correa H, Southard-Smith EM. Enteric neuron imbalance and proximal dysmotility in ganglionated intestine of the Sox10Dom ${ }^{+}$Hirschsprung mouse model. Cell Mol Gastroenterol Hepatol. 2015;1(1):87-101.

37. Musser MA, Michelle Southard-Smith E. Balancing on the crest - Evidence for disruption of the enteric ganglia via inappropriate lineage segregation and consequences for gastrointestinal function. Dev Biol. 2013;382(1):356-364

38. Wang $\mathrm{H}$, et al. The timing and location of glial cell line-derived neurotrophic factor expression determine enteric nervous system structure and function. J Neurosci. 2010;30(4):1523-1538.

39. Viader A, Wright-Jin EC, Vohra BP, Heuckeroth RO, Milbrandt J. Differential regional and subtype-specific vulnerability of enteric neurons to mitochondrial dysfunction. PLoS ONE. 2011;6(11):e27727.

40. $\mathrm{Li} \mathrm{Z}$, et al. Essential roles of enteric neuronal serotonin in gastrointestinal motility and the development/survival of enteric dopaminergic neurons. J Neurosci. 2011;31(24):8998-9009.

41. Chalazonitis A, et al. Bone morphogenetic protein regulation of enteric neuronal phenotypic diversity: relationship to timing of cell cycle exit. J Comp Neurol. 2008;509(5):474-492.

42. Soret R, et al. Short-chain fatty acids regulate the enteric neurons and control gastrointestinal motility in rats. Gastroenterology. 2010;138(5):1772-1782.

43. Fung C, Unterweger P, Parry LJ, Bornstein JC, Foong JP. VPAC1 receptors regulate intestinal secretion and muscle contractility by activating cholinergic neurons in guinea pig jejunum. Am J Physiol Gastrointest Liver Physiol. 2014;306(9):G748-G758.

44. Chandrasekharan B, Nezami BG, Srinivasan S. Emerging neuropeptide targets in inflammation: NPY and VIP. Am J Physiol Gastrointest Liver Physiol. 2013;304(11):G949-G957.

45. Lelievre V, et al. Gastrointestinal dysfunction in mice with a targeted mutation in the gene encoding vasoactive intestinal polypeptide: a model for the study of intestinal ileus and Hirschsprung's disease. Peptides. 2007;28(9):1688-1699.

46. Cobos I, Borello U, Rubenstein JL. Dlx transcription factors promote migration through repression of axon and dendrite growth. Neuron. 2007;54(6):873-888

47. Mowat DR, Wilson MJ, Goossens M. Mowat-Wilson syndrome. J Med Genet. 2003;40(5):305-310.

48. Le TN, et al. GABAergic interneuron differentiation in the basal forebrain is mediated through direct regulation of glutamic acid decarboxylase isoforms by $D l x$ homeobox transcription factors. $J$ Neurosci. 2017;37(36):8816-8829.

49. Pla R, et al. D1x1 and Dlx2 promote interneuron GABA synthesis, synaptogenesis, and dendritogenesis. Cereb Cortex. 2018;28(11):3797-3815.

50. El-Chammas K, Sood MR. Chronic intestinal pseudo-obstruction. Clin Colon Rectal Surg. 2018;31(2):99-107.

51. D'Autréaux F, et al. Expression level of Hand2 affects specification of enteric neurons and gastrointestinal function in mice. Gastroenterology. 2011;141(2):576-587.

52. Pichel JG, et al. Defects in enteric innervation and kidney development in mice lacking GDNF. Nature. 1996;382(6586):73-76

53. Schuchardt A, D'Agati V, Larsson-Blomberg L, Costantini F, Pachnis V. Defects in the kidney and enteric nervous system of mice lacking the tyrosine kinase receptor Ret. Nature. 1994;367(6461):380-383.

54. Cacalano G, et al. GFRalpha1 is an essential receptor component for GDNF in the developing nervous system and kidney. Neuron. 1998;21(1):53-62.

55. Enomoto H, Crawford PA, Gorodinsky A, Heuckeroth RO, Johnson EM, Milbrandt J. RET signaling is essential for migration, 
axonal growth and axon guidance of developing sympathetic neurons. Development. 2001;128(20):3963-3974.

56. Creedon DJ, et al. Neurturin shares receptors and signal transduction pathways with glial cell line-derived neurotrophic factor in sympathetic neurons. Proc Natl Acad Sci USA. 1997;94(13):7018-7023.

57. Wright-Jin EC, Grider JR, Duester G, Heuckeroth RO. Retinaldehyde dehydrogenase enzymes regulate colon enteric nervous system structure and function. Dev Biol. 2013;381(1):28-37.

58. Carpenter AE, et al. CellProfiler: image analysis software for identifying and quantifying cell phenotypes. Genome Biol. 2006;7(10):R100

59. Tabara H, et al. Neonatal lethality in knockout mice expressing the kinase-dead form of the gefitinib target GAK is caused by pulmonary dysfunction. PLoS ONE. 2011;6(10):e26034.

60. López SH, et al. Loss of Tbx3 in murine neural crest reduces enteric glia and causes cleft palate, but does not influence heart development or bowel transit. Dev Biol. 2018;444 suppl 1:S337-S351.

61. Dobin A, et al. STAR: ultrafast universal RNA-seq aligner. Bioinformatics. 2013;29(1):15-21.

62. Lahens NF, et al. A comparison of Illumina and Ion Torrent sequencing platforms in the context of differential gene expression. BMC Genomics. 2017;18(1):602.

63. Ritchie ME, et al. limma powers differential expression analyses for RNA-sequencing and microarray studies. Nucleic Acids Res 2015;43(7):e47

64. Law CW, Chen Y, Shi W, Smyth GK. voom: Precision weights unlock linear model analysis tools for RNA-seq read counts. Genome Biol. 2014;15(2):R29. 